Please cite as

A grand-potential based phase-field approach for simulating growth of intermetallic phases in multicomponent alloy systems

Sourav Chatterjee, Nele Moelans

Accepted for Acta Materialia (2021).

- Doi : $10.1016 /$ j.actamat.2021.116630 


\title{
A grand-potential based phase-field approach for simulating growth of intermetallic phases in multicomponent alloy systems
}

\author{
Sourav Chatterjee ${ }^{a, \star}$, Nele Moelans ${ }^{a}$ \\ ${ }^{a}$ Department of Materials Engineering, KU Leuven, Kasteelpark Arenberg 44, \\ Leuven BE-3001, Belgium \\ ${ }^{*}$ Corresponding author. E-mail addresses: \\ sourav.chatterjee@kuleuven.be (S. Chatterjee), nele.moelans@kuleuven.be (N. \\ Moelans)
}


Intermetallic phase-based alloys, in particular transition-metal aluminides, are potential structural and coating materials for high-temperature applications. Such applications usually involve interdiffusion between two dissimilar materials. To simulate interdiffusion microstructures quantitatively, it becomes essential to solve alloy phase-field models in conjunction with multicomponent CALPHAD databases. This coupling, however, still remains a challenge when considering binary or multicomponent intermetallic phases. Here, a novel method that incorporates successfully diffusion potential dependent-properties of bulk multicomponent phases into a grand-potential based multi-phase-field model is proposed. It uses phase-specific properties directly precomputed from CALPHAD-type databases as discrete functions of solute diffusion potentials. Six different alloy cases, ranging from a five-phase binary (Ni-Al) to a two-phase quaternary (Al-Cr-Ni-Fe) alloy, are simulated to illustrate the application and correctness of the method. The cases include both substitutional and intermetallic phases. Where a comparison is possible, the simulations show good agreement with DICTRA and experimental results, thus validating our proposed method. In contrast to our approach, we find that DICTRA fails in three of the simulations involving ordered intermetallics. We further show that the interface width in this model can be varied without accuracy loss, thus enabling computationally affordable simulations at experimentally comparable length and time scales.

Keywords: Intermetallic phases; Phase transformation; Multicomponent diffusion; Microstructre; Growth kinetics. 


\section{Introduction}

Several technologically relevant alloys, e.g. Ni-base superalloys, complex concentrated alloys [1], consist of intermetallic phases. These solid phases generally form and grow by interdiffusion between two adjoining dissimilar multicomponent alloys. Due to their high melting point, high specific strength, and good oxidation resistance, these phases are critical for joint performance at elevated temperatures. Consequently, optimization of their microstructure and growth through alloy additions is desirable for improved joint performance. Experimental approaches to achieve this necessitate longer testing times. Moreover, analytical treatments of their growth always require simplifying assumptions. Therefore, computational tools, like DICTRA (DIffusion-Controlled TRAnsformation) software [2], are indispensable approaches for optimizing their growth and microstructure.

For reliable simulations, DICTRA provides a systematic approach of integrating multicomponent CALPHAD (CALculation of PHAse Diagram)type databases into simulations [2]. However, a major drawback of this software is that it is only possible to simulate simple one-dimensional geometry. As a result, it cannot take account of the morphology of phases. Besides DICTRA, phase-field methods have also evolved into a computational tool for simulating the growth of multicomponent phases [3-27]. In contrast to DICTRA, which treats the interface as sharp, in a phase-field model the properties vary smoothly in the interfacial region [28], thus enabling to simulate complex morphologies. Unfortunately, alloy phase-field models still lack a consistent approach of coupling with CALPHAD-based databases. 
In the phase-field model proposed by Wheeler et al. [3] for binary alloys, a coupling can be realized by directly employing the composition-dependent molar Gibbs energy of the bulk phases into the (Helmholtz) free energy functional [17-22]. In this model, however, it becomes computationally expensive to perform accurate simulations at the micrometer length scales $(\geq 10 \mu \mathrm{m})$, regardless of bulk properties (see Refs [6], [8], [9], [13], \& [29], for details), thus precluding quantitative comparison of simulated microstructures with experiments at these length scales.

Two approaches have been proposed so far that enable quantitatively accurate simulations at the micrometer length scale [13]. In the first approach, the evolution equations for phase-field(s) and solute composition(s) are derived from minimization of a (Helmholtz) free energy functional [5], [8], [11], [14]. While in the second approach, the evolution equations for phase-field(s) and solute diffusion potential(s) are derived from minimization of a grandpotential functional [13], [15]. As pointed out by Plapp [13], both approaches are equivalent in terms of the evolution equations.

However, from the viewpoint of coupling with CALPHAD-type databases, two aspects distinguish the two aforementioned approaches. First, concerning the solute phase mole fractions [5], [8]. For the sake of concreteness, consider a two-phase, $\alpha+\beta$, binary A-B alloy. Then, the condition of equality of solute phase diffusion potentials [8] (or "quasiequilibrium" condition [11]) requires that: $\tilde{\mu}_{B}^{\alpha}\left(X_{B}^{\alpha}\right)=\tilde{\mu}_{B}^{\beta}\left(X_{B}^{\beta}\right)$, where $\tilde{\mu}_{B}^{\alpha}\left(X_{B}^{\alpha}\right)$ and $\tilde{\mu}_{B}^{\beta}\left(X_{B}^{\beta}\right)$ are the solute phase diffusion potentials of phases $\alpha$ and $\beta$, respectively. In the Helmholtz approach, to calculate the two local solute phase mole fractions, $X_{B}^{\alpha}$ and $X_{B}^{\beta}$, the equation enforcing the equality of phase diffusion potentials has to be 
solved together with an auxiliary equation and the evolution equations at each grid point and time step [8]. Furthermore, either nonlinear iterative schemes [10], [29], [30] or linear approximations of diffusion potentials [31] are required to solve this nonlinear equation for non-dilute solutions. Nevertheless, in a generalized formulation [11], [14], consisting of $n$ components and $p$ phases, the number of such conditions required are $(n-1)(p-1)$. Contrarily, if the evolution equations follow from a grand-potential functional, then these conditions are not needed. Since then the solute phase mole fractions are expressed explicitly as functions of solute diffusion potentials [13]. Second, as presented in Table 1, in the (Helmholtz) free energy based approach all bulk properties are explicit functions of solute phase mole fractions [23-27, 31] while in the grand-potential based approach all bulk properties are explicit functions of solute diffusion potentials [13]. It is also evident from this table that both approaches require the same number of thermodynamic properties per phase for a given alloy system. Notably, although composition-dependent bulk properties can be calculated from databases using software packages, like ThermoCalc [32], no such direct method is available to evaluate diffusion potential-dependent bulk properties [33]. Thus limiting the application of the latter approach for "real" alloys.

The reason for the unavailability of a direct calculation method is that the phase mole fraction of a solute $k, X_{k}^{\theta}(\tilde{\boldsymbol{\mu}})$, expressed as an explicit function of solute diffusion potentials is first needed and then only the molar grandpotential $\Omega_{m}^{\theta}(\tilde{\boldsymbol{\mu}})$ of an arbitrary phase $\theta$ can be determined [13]. However for non-dilute substitutional phases, even if an analytical expression for solute diffusion potential $\tilde{\mu}_{k}^{\theta}\left(\mathbf{X}^{\theta}\right)$ as a function of solute phase mole fraction(s) is 
known, it is still analytically intractable to calculate the inverse relation between mole fraction and diffusion potential, that is $X_{k}^{\theta}(\tilde{\boldsymbol{\mu}})[10]$. This explains why all previous works based on the grand-potential approach either assume dilute/ideal solution models [13], [15], [34], [35] or use simplified second-order polynomials of the molar Gibbs free energy $G_{m}^{\theta}\left(\mathbf{X}^{\theta}\right)$ [36-43]. Furthermore, the coefficients of these simplified polynomials are calculated either by Taylor expansion (proposed by Plapp [13] for binary alloys and later extended to multicomponent alloys by Choudhury et al. in [44]) or by least squares method [33], and remain valid only for "small" deviations from equilibrium values. However, it becomes increasingly infeasible to fit $G_{m}^{\theta}\left(\mathbf{X}^{\theta}\right)$ as a second-order polynomial in multicomponent multiphase alloys [27], [45], [46]. Furthermore, to our knowledge, grand-potential based approaches have not been linked with CALPHAD-based mobility databases so far.

Here, we introduce a computationally efficient method that incorporates the diffusion potential-dependent CALPHAD properties into a grand-potential based multi-phase-field model. In Section 2, the phase-field model is formulated starting from a grand-potential functional, following the approach of Plapp [13], with the difference that the diffusion equation(s) are solved in terms of evolution of solute mole fraction(s) rather than solute diffusion potential(s). The prerequisite thermodynamic and kinetic properties are also detailed in this section. The application of the method is illustrated for six different (non-dilute and non-ideal) alloys in Section 3, and the results are concluded in Section 4. 


\section{Formulation and precomputed properties}

\subsection{Functional and molar grand-potential}

Consider an isothermal-isobaric inhomogeneous system consisting of $p$ phases and $n$ components occupying a constant volume $V$. Let $\boldsymbol{\phi}(\mathbf{x}, t)=\left\{\phi_{\theta=1 \ldots p}\right\}$ denote the set of $p$ phase-field variables at any spatial point $\mathbf{x}$ and time $t$. The phase-field variable $\phi_{\theta}$ varies spatially such that $\phi_{\theta}=1$ at bulk phase $\theta$ and $\phi_{\theta}$ $=0$ at any phase $p \neq \theta$. Also, let the set of $(n-1)$ independent solute diffusion potentials be denoted by $\tilde{\boldsymbol{\mu}}(\mathbf{x}, t)=\left\{\tilde{\mu}_{k=1 \ldots(n-1)}\right\}$. The diffusion potential variable of a solute component $k$ is defined as $\tilde{\mu}_{k}=\left(\mu_{k}-\mu_{n}\right)[\mathrm{J} / \mathrm{mol}]$, which is the difference between the chemical potentials of a solute component $k$ and a solvent component $n$. Since the independent thermodynamic variables are the $(n-1)$ solute diffusion potentials, the functional to be minimized is the grand-potential $\Omega$ of the system [13], [15] and is given by

$$
\Omega[\boldsymbol{\phi}, \tilde{\boldsymbol{\mu}}]=\int_{V}\left[\omega_{b u l k}(\boldsymbol{\phi}, \tilde{\boldsymbol{\mu}})+\omega_{i n t}(\boldsymbol{\phi}, \nabla \boldsymbol{\phi})\right] d v(\mathbf{x})
$$

where the bulk grand-potential density, $\omega_{\text {bulk }}(\phi, \tilde{\boldsymbol{\mu}})$, and interfacial energy density, $\omega_{\text {int }}(\boldsymbol{\phi}, \nabla \boldsymbol{\phi})$, contributions in Eq. (1) take the following forms:

$$
\begin{aligned}
\omega_{b u l k}(\boldsymbol{\phi}, \tilde{\boldsymbol{\mu}}) & =\sum_{\theta=1}^{p} h_{\theta}(\boldsymbol{\phi}) \omega_{\theta}(\tilde{\boldsymbol{\mu}}) \\
\omega_{\text {int }}(\boldsymbol{\phi}, \nabla \boldsymbol{\phi}) & =(1 / 2) \kappa|\nabla \boldsymbol{\phi}|^{2}+m g(\boldsymbol{\phi}) .
\end{aligned}
$$

Here, $\omega_{\theta}(\tilde{\boldsymbol{\mu}})\left[\mathrm{J} / \mathrm{m}^{3}\right]$ is the bulk grand-potential density of a phase $\theta ; \kappa[\mathrm{J} / \mathrm{m}]$ is the gradient energy coefficient; and $m\left[\mathrm{~J} / \mathrm{m}^{3}\right]$ is the barrier height. The 
interpolation function, $h_{\theta}(\phi)$, in Eq. (2) and the multi-well function, $g(\phi)$, in Eq. (3) are taken from Ref. [14]

$$
\begin{aligned}
h_{\theta}(\boldsymbol{\phi}) & =\frac{\phi_{\theta}^{2}}{\sum_{\theta=1}^{p} \phi_{\theta}^{2}}, \\
g(\boldsymbol{\phi}) & =\sum_{\theta=1}^{p}\left[(1 / 4) \phi_{\theta}^{4}-(1 / 2) \phi_{\theta}^{2}\right]+(1.5 / 2) \sum_{\theta=1}^{p} \sum_{\sigma=1}^{p} \phi_{\theta}^{2} \phi_{\sigma}^{2}+(1 / 4) .
\end{aligned}
$$

Further, as shown in Refs. [8], [13] [14], the two constant parameters $\kappa$ and $m$ in Eq. (3) can be calculated for a given choice of interfacial energy $\sigma_{\alpha \beta}$ and interfacial thickness $l_{\alpha \beta}$ using the following relations:

$$
\kappa=(3.0 / 4.0) \sigma_{\alpha \beta} l_{\alpha \beta}, \quad m=6.0\left(\sigma_{\alpha \beta} / l_{\alpha \beta}\right),
$$

irrespective of bulk properties. Assuming constant molar volume $V_{m}\left[\mathrm{~m}^{3} / \mathrm{mol}\right]$, the grand-potential density $\omega_{\theta}(\tilde{\boldsymbol{\mu}})$ in Eq. (2) is related to the molar grandpotential $\Omega_{m}^{\theta}[\mathrm{J} / \mathrm{mol}]$ by $\omega_{\theta}(\tilde{\boldsymbol{\mu}})=\Omega_{m}^{\theta}(\tilde{\boldsymbol{\mu}}) / V_{m}$. The property $\Omega_{m}^{\theta}$ is here defined as the Legendre transform of the molar Gibbs energy $G_{m}^{\theta}[\mathrm{J} / \mathrm{mol}]$ that replaces mole fractions with diffusion potentials as the independent variables,

$$
\Omega_{m}^{\theta}(\tilde{\boldsymbol{\mu}})=G_{m}^{\theta}\left(\mathbf{X}^{\theta}(\tilde{\boldsymbol{\mu}})\right)-\sum_{k=1}^{n-1} \tilde{\mu}_{k} X_{k}^{\theta}(\tilde{\boldsymbol{\mu}})
$$

Differentiating Eq. (6) and using the relation: $d G_{m}^{\theta}=\sum_{k=1}^{n-1} \tilde{\mu}_{k} d X_{k}^{\theta}$, it follows that the phase mole fraction of a solute $k$ in phase $\theta$ is $X_{k}^{\theta}(\tilde{\boldsymbol{\mu}})=-\partial \Omega_{m}^{\theta} / \partial \tilde{\mu}_{k}$. It is further noted that the molar grand-potential $\Omega_{m}^{\theta}(\tilde{\boldsymbol{\mu}})$ of a general solution phase can be computed indirectly using Eq. (6), provided $X_{k}^{\theta}(\tilde{\boldsymbol{\mu}})$ is known (either analytically or numerically) as a function of diffusion potentials. 


\subsection{Evolution equations}

The first variation in the system grand-potential functional $\Omega$, Eq. (1), with respect to (w.r.t) the variables $\phi$ and $\tilde{\boldsymbol{\mu}}$ yields the following set of equations

$$
\begin{aligned}
& \frac{\delta \Omega}{\delta \tilde{\mu}_{k}}=X_{k}(\tilde{\boldsymbol{\mu}}, \boldsymbol{\phi})=\sum_{\theta=1}^{p} h_{\theta}(\boldsymbol{\phi}) X_{k}^{\theta}(\tilde{\boldsymbol{\mu}}), \\
& \frac{\delta \Omega}{\delta \phi_{\theta}}=m \frac{\partial g(\boldsymbol{\phi})}{\partial \phi_{\theta}}-\kappa \Delta \phi_{\theta}+\sum_{\substack{\sigma=1 \\
\sigma \neq \theta}}^{p-1} \frac{\partial h_{\sigma}}{\partial \phi_{\theta}} \frac{\left\{\Omega_{m}^{\sigma}(\tilde{\boldsymbol{\mu}})-\Omega_{m}^{\theta}(\tilde{\boldsymbol{\mu}})\right\}}{V_{m}},
\end{aligned}
$$

where $\Delta$ is the laplacian and $X_{k}(\tilde{\boldsymbol{\mu}}, \phi)$ is the conserved overall mole fraction of a solute $k$. The term in the brackets divided by molar volume in Eq. (8) is what Plapp [13] refers to as the "thermodynamic" driving force for phase change, which is the difference between the grand-potential densities. It thus follows from Eq. (8) that the phase-field evolves as [47]

$$
\frac{\partial \phi_{\theta}(\mathbf{x}, t)}{\partial t}=-L_{\phi} \frac{\delta \Omega}{\delta \phi_{\theta}}
$$

where $L_{\phi}\left[\mathrm{m}^{3} / \mathrm{Js}\right]$ is phase-field mobility. Additionally, the local form of mass balance for an independent solute component $k$ takes the form [48]

$$
\frac{\partial c_{k}(\mathbf{x}, t)}{\partial t}=-\nabla \mathbf{j}_{k}^{\#}=\nabla\left[\sum_{j=1}^{n-1} \frac{L_{k j}^{n}(\tilde{\boldsymbol{\mu}}, \boldsymbol{\phi})}{V_{m}} \nabla \tilde{\mu}_{j}(\mathbf{x}, t)\right]
$$

where $c_{k}(\mathbf{x}, t)\left[\mathrm{mol} / \mathrm{m}^{3}\right]$ is the overall molar density of solute component $k ; \mathbf{j}_{k}^{\#}$ $\left[\mathrm{mol} /\left(\mathrm{m}^{2} \mathrm{~s}\right)\right]$ is the flux of diffusing component $k$; and $L_{k j}^{n}(\tilde{\boldsymbol{\mu}}, \boldsymbol{\phi})\left[\left(\mathrm{mol} \mathrm{m}{ }^{2}\right) / \mathrm{Js}\right]$ are the coefficients of the overall Onsager matrix relating the diffusing flux of solute $k$ to the driving force due to gradient in diffusion potential of solute $j$. 
Here, the superscript $n$ is the dependent solvent component and \# denotes the number-fixed frame [48]. Equivalently, Eq. (10) can be written in terms of the solute mole fractions as follows (see Appendix A):

$$
\begin{aligned}
& \frac{\partial X_{k}(\mathbf{x}, t)}{\partial t}=\nabla\left[\sum_{r=1}^{n-1} D_{k r}^{n}(\tilde{\boldsymbol{\mu}}, \phi) \nabla X_{r}(\mathbf{x}, t)\right] \\
& -\nabla\left[\sum_{r=1}^{n-1} D_{k r}^{n}(\tilde{\boldsymbol{\mu}}, \boldsymbol{\phi}) \sum_{\substack { \theta=1 \\
\begin{subarray}{c}{\sigma=1 \\
\sigma \neq \theta{ \theta = 1 \\
\begin{subarray} { c } { \sigma = 1 \\
\sigma \neq \theta } }\end{subarray}}^{p-1} \frac{\partial h_{\sigma}}{\partial \phi_{\theta}}\left\{X_{r}^{\sigma}(\tilde{\boldsymbol{\mu}})-X_{r}^{\theta}(\tilde{\boldsymbol{\mu}})\right\} \nabla \phi_{\theta}(\mathbf{x}, t)\right],
\end{aligned}
$$

where $D_{k r}^{n}(\tilde{\boldsymbol{\mu}}, \boldsymbol{\phi})=\left(\sum_{j=1}^{n-1} L_{k j}^{n}(\tilde{\boldsymbol{\mu}}, \boldsymbol{\phi})\left[\partial X_{j}(\tilde{\boldsymbol{\mu}}, \boldsymbol{\phi}) / \partial \tilde{\mu}_{r}\right]^{-1}\right)\left[\mathrm{m}^{2} / \mathrm{s}\right]$ are the coefficients of the overall chemical diffusivity matrix; expressed as the product of the overall Onsager matrix and the inverse of the overall susceptibility matrix $\left[\partial X_{j}(\tilde{\boldsymbol{\mu}}, \phi) / \partial \tilde{\mu}_{r}\right]^{-1}$. As introduced in [13], [15], the coefficients of the overall susceptibility matrix can be determined (for a convex Gibbs energy) by differentiating Eq. (7) w.r.t the diffusion potential variable, i.e.,

$$
\frac{\partial X_{j}(\boldsymbol{\phi}, \tilde{\boldsymbol{\mu}})}{\partial \tilde{\mu}_{r}}=\sum_{\theta=1}^{p} h_{\theta}(\phi) \chi_{j r}^{\theta}(\tilde{\boldsymbol{\mu}}),
$$

where $\chi_{j r}^{\theta}(\tilde{\boldsymbol{\mu}})=\partial X_{j}^{\theta}(\tilde{\boldsymbol{\mu}}) / \partial \tilde{\mu}_{r}[\mathrm{~mol} / \mathrm{J}]$ are the coefficients of the susceptibility matrix of a bulk phase $\theta$ as a function of diffusion potentials. The phase susceptibility matrix $\chi_{j r}^{\theta}(\tilde{\boldsymbol{\mu}})$ can be calculated by inverting the thermodynamic factor matrix $\Theta_{k j}^{\theta}=\partial \tilde{\mu}_{k}^{\theta} / \partial X_{j}$. It can be shown that for a binary two-phase alloy Eq. (11) reduces to Eq. (33) in Ref. [8]. The coefficients of the overall Onsager matrix $L_{k j}^{n}(\tilde{\boldsymbol{\mu}}, \phi)$ in Eq. (10) are assumed to be a weighted sum of the bulk coefficients of the Onsager matrix $L_{k j}^{n \theta}(\tilde{\boldsymbol{\mu}})$ of each distinct phases 
within the system, i.e.,

$$
L_{k j}^{n}(\tilde{\boldsymbol{\mu}}, \phi)=\sum_{\theta=1}^{p} h_{\theta}(\phi) L_{k j}^{n \theta}(\tilde{\boldsymbol{\mu}})
$$

As derived in Refs. [12], [14], the phase-field mobility $L_{\phi}$ can be calculated using the following relation

$$
L_{\phi}^{e q}=4 m /(3 \kappa \zeta)
$$

where $\zeta=\sum_{k=1}^{n-1}\left(X_{k}^{\theta, e q}-X_{k}^{\sigma, e q}\right) \sum_{j=1}^{n-1}\left(L_{k j}^{n \theta, e q} V_{m}\right)^{-1}\left(X_{j}^{\theta, e q}-X_{j}^{\sigma, e q}\right)[12]$. The parameter $\zeta$ has units of $\left[\mathrm{Js} / \mathrm{m}^{5}\right]$. Hence, together with initial and boundary conditions, solving for the set of field variables $\left\{\tilde{\mu}_{k}(\mathbf{x}, t), \phi_{\theta}(\mathbf{x}, t), X_{k}(\mathbf{x}, t)\right\}$ using Eqs. (7), (9) and (10) completes the model formulation. In this work, we do not solve for the evolution equation of the diffusion potentials (see Appendix A), as formulated in [13], [15], [35-38, 40-42]. Since it is convenient to initialize the simulation with mole fraction(s) rather than diffusion potential(s), we solve for the diffusion potential(s) and mole fraction(s) variables implicitly using the coupled Eqs. (7) and (10), respectively. This approach is based on the work of Kim et al. [8].

\subsection{Precomputed properties}

There is no in-built command in ThermoCalc to determine the molar grandpotential $\Omega_{m}^{\theta}(\tilde{\boldsymbol{\mu}})$. To calculate this quantity indirectly using Eq. (6), the inverse relation between the phase mole fraction and diffusion potential, i.e., $X_{k}^{\theta}(\tilde{\boldsymbol{\mu}})$, is first required. In theory, this relation can always be calculated for 
a convex Gibbs energy. But in practice, it cannot be determined analytically for non-dilute and non-ideal (binary or multicomponent) solution phases, as discussed in the Introduction. To find this numerically, we performed a minimization w.r.t the (intensive) diffusion potential(s) variables(s) instead of the (extensive) composition(s) variable(s) during the precomputation stage. This minimization was achieved in the TC-Toolbox for MATLAB. Consequently, the phase mole fraction $X_{k}^{\theta}(\tilde{\boldsymbol{\mu}})$ of an independent solute $k$ in a phase was calculated via an in-built command in TC-Toolbox.

Next, the molar Gibbs free energy $G_{m}^{\theta}$ as a discrete function of solute diffusion potential(s) was calculated using an in-built command. Subsequently, the molar grand-potential was indirectly calculated from Eq. (6) by using the precomputed solute phase mole fraction(s) and the Gibbs energy. To further verify the indirect approach, the following direct approach was taken. It is straightforward to show that the molar grand-potential $\Omega_{m}^{\theta}(\tilde{\boldsymbol{\mu}})$ of a phase is equal to the chemical potential of the dependent component $\mu_{n}^{\theta}(\tilde{\boldsymbol{\mu}})$ in that phase (see Eqs. (1a)-(1d) in Ahmad et al. [7], Eqs. (29)-(30) in Eiken et al. [11] and Eq.(B.1)). As a result, the molar grand-potential was also numerically computed by retrieving the chemical potential of the dependent component. A comparison of the data obtained via the two approaches confirmed the fact that $\Omega_{m}^{\theta}(\tilde{\boldsymbol{\mu}})=\mu_{n}^{\theta}(\tilde{\boldsymbol{\mu}})$ (Appendix B). Henceforth, we will use these two identical terms interchangeably. It is thus clear that the difference in the chemical potentials between the adjacent phases, $\Delta \Omega_{m}^{\theta \rightarrow \sigma}=\Delta \mu_{n}^{\theta \rightarrow \sigma}=\left(\mu_{n}^{\sigma}-\mu_{n}^{\theta}\right)$, drives the phase change in this model [11].

Similarly, the independent coefficients of the thermodynamic factor matrix $\Theta_{k j}^{\theta}(\tilde{\boldsymbol{\mu}})$ were precomputed using an in-built command in TC-toolbox, and 
then inverted to calculate the coefficients of the solute susceptibility matrix $\chi_{k j}^{\theta}(\tilde{\boldsymbol{\mu}})$. The independent coefficients of the Onsager matrix $L_{k j}^{n \theta}(\tilde{\boldsymbol{\mu}})$ were first calculated by minimizing w.r.t the numerically computed solute phase mole fraction(s), $X_{k}^{\theta}(\tilde{\boldsymbol{\mu}})$, using an in-built command. Then these coefficients were tabulated as functions of solute diffusion potential(s). The interested reader can refer to Supplementary materials S5 and S6 for the MATLAB scripts detailing the procedure and in-built commands used to precompute these properties for a ternary phase. To conclude this section, the three thermodynamic properties and one kinetic property needed beforehand are stated: i) the molar grand-potential $\Omega_{m}^{\theta}$, ii) the phase mole fractions $X_{k}^{\theta}$, iii) the solute susceptibility matrix $\chi_{i j}^{\theta}$, and iv) the Onsager diffusion matrix $L_{k j}^{n \theta}$. Furthermore, the computation tool for phase-field simulations is developed using MOOSE [49], [30]. The implementation details and the simulation parameters are given in Supplementary material S1 and Supplementary Material S3, respectively.

\section{$3 \quad$ Results and discussions}

\subsection{Five-phase and two-phase $\mathrm{Ni}$-Al alloys}

First, a five-phase Ni-Al alloy was selected for simulating the growth of binary intermetallic phases. Assuming all equilibrium phases are present, the $\mathrm{Ni} / \mathrm{Al}$ diffusion couple studied at $1000 \mathrm{~K}$ consists of three intermetallic phases: $\gamma^{\prime}-\mathrm{Ni}_{3} \mathrm{Al}\left(\mathrm{L}_{2}\right), \mathrm{NiAl}(\mathrm{B} 2), \& \mathrm{Al}_{3} \mathrm{Ni}_{2}\left(\mathrm{D} 5_{13}\right)$ and two substitutional phases: $\gamma$ and liquid (Fig.1a). The equilibrium Al-mole fractions at the four 
two-phase regions, i.e., $\gamma / \gamma^{\prime}, \gamma^{\prime} / \mathrm{NiAl}, \mathrm{NiAl} / \mathrm{Al}_{3} \mathrm{Ni}_{2}$ and $\mathrm{Al}_{3} \mathrm{Ni}_{2} /$ liquid, are $0.1246 / 0.2372,0.2746 / 0.4065,0.5414 / 0.6002$ and $0.6802 / 0.8931$ (marked as a to $\mathrm{h}$ in Fig.1a). Here, $\mathrm{Al}$ is the independent solute component and $\mathrm{Ni}$ is the dependent solvent component. Although this choice is arbitrary, its relevance is that the molar grand-potential of these phases were numerically computed by retrieving the chemical potential of $\mathrm{Ni}$. The procedure to determine the $\mathrm{Al}$ diffusion potential interval, within which the prerequisite material properties were precomputed, is detailed in Appendix 5.1.

The length of the simulated one-dimensional diffusion couple was $300 \mu \mathrm{m}$. The initial layer thickness of $\mathrm{Ni}_{3} \mathrm{Al}, \mathrm{NiAl}$ and $\mathrm{Al}_{3} \mathrm{Ni}_{2}$ phases were $20 \mu \mathrm{m}, 10$ $\mu \mathrm{m}$ and $10 \mu \mathrm{m}$, respectively. Fig.1b shows how the phase-field simulation of Al-mole fraction profiles varies with time for three different interface widths. The simulations show that the $\beta$-NiAl phase grows much faster in comparison to $\mathrm{Al}_{3} \mathrm{Ni}_{2}$ and $\mathrm{Ni}_{3} \mathrm{Al}$ phases, and the simulated mole fraction profiles are independent of the choice of interface width $l_{\alpha \beta}$ (Fig.1b). As anticipated for a diffusion-controlled process, the growth kinetics of $\mathrm{NiAl}$ and $\mathrm{Al}_{3} \mathrm{Ni}_{2}$ follows a parabolic growth law (Fig.1c). The growth constants of $\beta-\mathrm{NiAl}$ and $\mathrm{Al}_{3} \mathrm{Ni}_{2}$ phases determined after fitting are shown in Fig.1c. Further, the simulation results indicate that the layer thickness of $\gamma^{\prime}-\mathrm{Ni}_{3} \mathrm{Al}$ phase decreases until $t<2.25 \mathrm{e} 4 \mathrm{hrs}$, and then subsequently grows linearly with square root of time (Fig.1c). Although the accuracy of the one-dimensional simulations remain unaltered by the choice of $l_{\alpha \beta}$, the simulation with $l_{\alpha \beta}=0.3 \mu \mathrm{m}$ requires about 9.6 times higher computation time compared to the simulation with $l_{\alpha \beta}=1.5 \mu \mathrm{m}$ (Supplementary material S2). Fig.1d shows a two dimensional simulated micrograph of the $\mathrm{Al}$ mole fraction field at $t=7 \mathrm{e} 4 \mathrm{hrs}$. 
This simulation could not compared with DICTRA, because the mobility data of $\mathrm{Al}_{3} \mathrm{Ni}_{2}$ phase was not available. However, we did try to validate by simulating the problem as a two-phase, $\gamma+\gamma^{\prime}$, system. However, DICTRA failed to simulate this problem for the same initial conditions as in the phasefield model. We therefore carried out another two-phase simulation at 1473 K. To compare with experimental results of Janssen [50], the initial Al mole fractions $\left(X_{A l}^{\gamma^{\prime}}=0.25\right.$ and $\left.X_{A l}^{\gamma}=10^{-4}\right)$ and the system length $(6 \mathrm{~mm})$ were set at the same values as those used by Campbell [51]. For this case, the relative percent $\mathrm{Al}$ saturation values in $\gamma$ and $\gamma^{\prime}$ phases are $-99.9456 \%$ and $8.352 \%$, respectively. The phase-field simulated results, that is the Ni-mole fraction and the $\gamma-\gamma^{\prime}$ interface displacement, show excellent agreement with DICTRA, for three different choice of interface widths (Figs.2a and 2b). At 43.2 hrs, the Ni-mole fraction profile also shows good quantitative agreement with the experimental data of Janssen [50] (Fig.2a). However, the phase-field (or the DICTRA) predicted growth constant is 1.275 times larger than the growth constant obtained after fitting the experimental data (Fig.2b). In order to test the Taylor approach [44], we performed another phase-field simulation with an interface width of $10 \mu \mathrm{m}$. Further, we assumed constant Al Onsager mobilities within each phase. Unsurprisingly, the growth constant obtained from the Taylor approach is 23.83 times that of the experimental growth constant (Fig.2b). The reason for this significant divergence from experimental data is a combination of two simplification: constant $\mathrm{Al}$ mobilities in each phase and the Taylor approximation of the molar grand-potential curves. This shows the advantage of the current coupling method over the method proposed by Choudhury et al. [44]. 


\subsection{Two-phase and three-phase Ni-Al-Cr alloys}

Next, two Ni-Al-Cr based couples were chosen at $1473 \mathrm{~K}$. Using the nominal compositions (Fig.3a) of the couples, the equilibrium solute mole fractions, as calculated from ThermoCalc, in the two-phase bcc/fcc and the threephase $\gamma$-fcc $/ \mathrm{L}_{2} / \mathrm{B} 2$ couples are $(0.870 \mathrm{Cr}, 0.013 \mathrm{Al}) /(0.367 \mathrm{Cr}, 0.1034 \mathrm{Al})$ and $(0.1466 \mathrm{Cr}, 0.1676 \mathrm{Al}) /(0.0758 \mathrm{Cr}, 0.2209 \mathrm{Al}) /(0.0676 \mathrm{Cr}, 0.2912 \mathrm{Al})$, respectively. Hereafter, the two diffusion couples are referred to as T1 and T2, respectively. In both couples, $\mathrm{Ni}$ is treated as the dependent solvent component while $\mathrm{Al}$ and $\mathrm{Cr}$ are the solute components. The diffusion potential intervals and the precomputed properties are detailed in Appendix 5.2.

The simulated length of both couples was taken to be $200 \mu \mathrm{m}$. In T1, the initial size of both the bcc and the fcc phases was $100 \mu \mathrm{m}$. At time $t=0$, the mole fractions of $\mathrm{Al}(\mathrm{Cr})$ in these phases were $9.39582 \times 10^{-4}(0.9543)$ and 0.0989 (0.3731). This equates to a relative percent Al-undersaturation (Crsupersaturation) of $92.516 \%(9.6425 \%)$ and $4.3536 \%(1.4768 \%)$, respectively. For three different choice of interface widths, the phase-field simulation of the $\mathrm{Cr}$ and $\mathrm{Al}$ mole fraction profiles in $\mathrm{T} 1$ alloy is in good agreement with the profiles calculated using DICTRA in the bulk phases (Fig.3b). For $t<20$ days, the variation in the position of the fcc/bcc boundary as a function of time shows a parabolic growth behaviour, and also agrees with the sharp-interface position calculated using DICTRA (Fig.4a).

In case of $\mathrm{T} 2$, the initial size of the $\gamma^{\prime}\left(\mathrm{L}_{2}\right)$ phase in the simulation was $34.0 \mu \mathrm{m}$. The initial $\mathrm{Al}(\mathrm{Cr})$-mole fractions in the $\gamma, \gamma^{\prime}$ and $\mathrm{B} 2$ phases are $0.1564(0.1598), 0.2159(0.0813)$ and 0.3008 (0.0523), which equates to 
an Al-saturation (Cr-saturation) of $-6.663 \%$ (9.004\%), $-2.261 \%(7.273 \%)$ and $3.305 \%$ (-22.533\%), respectively. For three different choice of interface widths, the phase-field simulation of the $\gamma-\gamma^{\prime}$ boundary indicate that it first grows into the $\gamma^{\prime}$ phase $(t=1 \mathrm{hr})$, and then shrinks back into the $\gamma$ phase ( $t=1$ day) (Fig.3c). While the $\gamma^{\prime}$-B2 phase boundary always migrates into the B2 phase with increasing time steps. For this reason, the layer width of the $\gamma^{\prime}$ phase initially decreases from $34.0 \mu \mathrm{m}$ to $30.22 \mu \mathrm{m}$ in $1.6925 \mathrm{hrs}$, and then subsequently grows to its final equilibrium thickness of $38.6467 \pm 0.012$ $\mu \mathrm{m}$ (Fig.4b). In the time interval $0.1<t<2.0$ days, the $\gamma^{\prime}$ phase thickens parabolically with time (inset in Fig.4b), and the growth constant $k_{p}$ is superimposed on Fig.4b. However for T2, the results could not be validated with DICTRA since it failed to simulate the problem for the same initial conditions as used in the phase-field model. Moreover, the simulation with $l_{\alpha \beta}=0.2 \mu \mathrm{m}$ requires nearly 67.4887 times of computation time compared to that of the simulation with $l_{\alpha \beta}=1.0 \mu \mathrm{m}$ (Supplementary material S2). For the same set of initial compositions used to perform the 1D simulation in alloy T2, Fig.3d shows the 2D simulated $\mathrm{Al}$ and $\mathrm{Cr}$ mole fraction fields at time $t=5$ days.

A two dimensional simulation of coarsening of $\gamma^{\prime}$ precipitates in a Al-Cr-Ni alloy at $1473 \mathrm{~K}$ was also performed. The interfacial energy $\sigma=6.9 \mathrm{~mJ} / \mathrm{m}^{2}$ was based on the value used by Grafe et al. [23]. The coarsening of $\gamma^{\prime}$ precipitates in a $\gamma$ matrix is shown in Figs.5b and 5c. Assuming LSW theory holds, the simulated cube of the mean radius as a function of time is fitted to a cubic growth law, and the result is shown in Fig.5d. To compare with mean-field models, the theoretically predicted growth constant was calculated 
based on Ref.[52] (see Supplementary material subsection S3.2, for details). We find that the growth constant obtained after fitting the simulated data is 4.536 times higher compared to the theoretical value (Fig.5d). Two possible explanations for this reasonable deviation are: i) the theory does not take into account the precipitate volume fraction and assumes it to be negligibly small, which consequently implies that there is no interaction between $\gamma^{\prime}$-precipitates, and ii) the diffusion potential (or composition) dependence of the mobility matrix is also not accounted for in the theory. As theoretically shown by Ardell (Table 1 in [53]) for binary alloys, the modified growth constant, when volume fractions is in the range of $0.06-0.15$, can be nearly $4-7$ times higher than that predicted by LSW theory. The $\gamma^{\prime}$ volume fraction in this simulation is around 0.12 .

\subsection{Two two-phase Ni-Al-Cr-Fe alloys}

Finally, we selected two two-phase Ni-Al-Cr-Fe diffusion couples at 1473

K. The nominal compositions of the two quaternary alloys (in mol\%) are Ni-4.8095Al-66.9577Cr-2.9959Fe and Ni-20.25Al-3.88Cr-4.71Fe. These quaternary diffusion couples are hereafter referred to as Q1 and Q2, respectively. In Q1 both phases (bcc and fcc) are substitutional solid solutions while couple Q2 involves an ordered intermetallic phase $\left(\gamma^{\prime}-\mathrm{L} 1_{2}\right)$. The equilibrium mole fractions in the bcc/fcc phases of Q1, as calculated from ThermoCalc, are $(0.0164 \mathrm{Al}, 0.8366 \mathrm{Cr}, 0.0288 \mathrm{Fe}) /(0.1088 \mathrm{Al}, 0.3497 \mathrm{Cr}, 0.0321 \mathrm{Fe})$. While in case of Q2, the equilibrium solute mole fractions in the $\gamma-\mathrm{fcc} / \gamma^{\prime}$ phases were calculated to be $(0.1671 \mathrm{Al}, 0.0526 \mathrm{Cr}, 0.0651 \mathrm{Fe}) /(0.2195 \mathrm{Al} 0.0322 \mathrm{Cr}, 0.0385 \mathrm{Fe})$. 
Similar to the ternary case, $\mathrm{Ni}$ is treated as the dependent solvent component while $\mathrm{Al}, \mathrm{Cr}$ and Fe are the solute components. The solute diffusion potential intervals and the precomputed properties are detailed in Appendix 5.3.

For both couples, the simulated length was set at $250 \mu \mathrm{m}$, and an initial phase size of $125 \mu \mathrm{m}$ was assumed. In case of Q1 alloy, the initial solute mole fractions in the bcc and the fcc phases were $(1.1898 \mathrm{e}-3 \mathrm{Al}, 0.9792 \mathrm{Cr}$, $0.0099 \mathrm{Fe})$ and $(0.0950 \mathrm{Al}, 0.36 \mathrm{Cr}, 0.05 \mathrm{Fe})$, respectively. This corresponds to a relative percent saturation of $(-92.7516 \% \mathrm{Al}, 17.0379 \% \mathrm{Cr},-65.5833 \% \mathrm{Fe})$ and $(-12.6474 \% \mathrm{Al}, 2.9314 \% \mathrm{Cr}, 55.5291 \% \mathrm{Fe})$ in the bcc and fcc phases, respectively. Within the bulk phases, the phase-field simulation of solute mole fraction profiles and those calculated using DICTRA shows reasonable agreement for two different choice of interface widths (Fig.6a). From the profiles, we observe that the predicted Fe mole fractions show deviation from the DICTRA calculated profiles at time $t=10$ days. More precisely, deviations of $2.032 \%$ at $155 \mu \mathrm{m}$ in fcc and $10.63 \%$ at $39 \mu \mathrm{m}$ in bcc were calculated. However, the simulated composition profiles completely agrees with DICTRA at the equilibrium state $(t=100$ days). This is also observed when the evolution of the diffuse fcc-bcc boundary position is compared to the sharp interface (Fig.7a). Using a lower tolerance limit, the simulated interface position was closer to the phase-field value but the computation time was significantly higher. For time $t<8$ days, the bcc phase thickens parabolically with time (inset in Fig.7a), and then gradually reaches towards the equilibrium state.

For couple Q2, the initial solute mole fractions in the fcc and the $\gamma^{\prime}$ phases were $(0.1860 \mathrm{Al}, 0.0492 \mathrm{Cr}, 0.0458 \mathrm{Fe})$ and $(0.2190 \mathrm{Al}, 0.0284 \mathrm{Cr}, 0.0484 \mathrm{Fe})$, 
which equates to a relative percent saturation of $(11.2846 \% \mathrm{Al},-6.4429 \% \mathrm{Cr}$, $-29.6721 \% \mathrm{Fe})$ and $(-0.2073 \% \mathrm{Al},-11.7708 \% \mathrm{Cr}, 25.850 \% \mathrm{Fe})$, respectively. Fig.6b shows the time evolution of the solute mole fractions as a function of length for three different choice of interface widths $l_{\alpha \beta}$. The migration of the fcc- $\gamma^{\prime}$ phase boundary is shown in Fig.7b. For time $t<8.4$ hrs., the quaternary $\gamma^{\prime}$ phase transforms to $\gamma$ phase parabolically with time (inset in

Fig.7b). By fitting the simulation data in this interval the growth constant $k_{p}$ is determined, and superimposed on Fig.7b. In contrast to Q1 alloy, however, we could not validate this simulation with DICTRA since it did not converge to a solution for the same initial conditions as used in the phase-field model. Although the accuracy of the simulated results are independent of the choice of the $l_{\alpha \beta}$ selected, the computation time with $l_{\alpha \beta}=0.25 \mu \mathrm{m}$ is almost 22.123 times that of the $l_{\alpha \beta}=0.75 \mu \mathrm{m}$ (Supplementary material S2).

\section{Conclusions}

In this paper, we have introduced a method to calculate properties such as molar grand-potential, phase mole fractions, solute susceptibilities etc. of multicomponent phases as functions of solute diffusion potentials directly from CALPHAD-based databases. Subsequently, we have utilized these prerequisite properties in a grand-potential based multi-phase-field model to simulate isothermal diffusional phase transformation. This coupling was implemented within a fully parallelized MOOSE-based application, and is independent of the commercial package, ThermoCalc, used in this work.

Further, the proposed method could be tested against DICTRA for simula- 
tions involving ternary (Al-Cr-Ni) and quaternary (Al-Cr-Ni-Fe) substitutional phases. The simulated results showed good quantitative agreement with DICTRA. Only for one out of the four simulations involving ordered intermetallic phases, a comparison with DICTRA was possible. When transformation between ordered phases was considered, we find that DICTRA fails in three of the simulations, for the same initial conditions and temperature as used in the phase-field model. Our method therefore provides an alternative approach to simulate diffusional transformation in ordered phases.

Moreover, we find that the accuracy of the phase-field simulated results does not vary for different choices of interface widths, thus enabling computationally feasible simulations at length and time scales comparable to bulk diffusion couple experiments. Also, simulations with higher interface width are computationally less costly compared to low interface width (one-thousandth of the simulation length) simulations. It is further shown that using the Taylor approximation of molar grand-potential combined with constant phase mobilities instead of actual CALPHAD based properties in simulations could induce significant errors in predicted growth constants.

Our current approach, however, suffers from a serious problem if extended to alloys constituted of five or more components. For instance, if a discretization of 75 equal parts is assumed, then for a quinary alloy the number of data points generated would be $75^{4}$. Moreover, it is worth noting that this limitation remains in phase-field approaches based on Helmholtz functional as well, since the number of prerequisite properties are same. Therefore, methods that reduce the data volume, while simultaneously maintaining the aceuracy of the required input data for arbitrary phases are needed, and will 
be explored in the future. Therefore, novel methods, for instance [54], that reduce data volume while simultaneously maintaining input data accuracy for arbitrary phases are needed, and will be explored in the future.

\section{$5 \quad$ Acknowledgments}

This work was supported by the European Research Council (ERC) under the European Union's Horizon 2020 research and innovation program (INTERDIFFUSION, grant agreement no. 714754). The computational resources and services used in this work were provided by the VSC (Flemish Supercomputer Center), funded by the Research Foundation - Flanders (FWO) and the Flemish Government - department EWI. S.C. thanks Yuri Coutinho from KU Leuven, for helping with MOOSE application and VSC cluster. Also, S.C. thanks Vincent Feyen from KU Leuven, for a fruitful discussion regarding the equality of molar grand-potential and chemical potential. 


\section{Tables and figures}

Table 1

Prerequisite properties for a ternary A-B-C alloy. Here, $G_{m}^{\theta}$ is the molar Gibbs free energy as functions of the independent phase molar fraction $X_{k}^{\theta}, \Omega_{m}^{\theta}$ is the molar grand-potential as function of the independent diffusion potentials $\tilde{\mu}_{k}=\left(\mu_{k}-\mu_{A}\right)$, $\left[\Theta_{k j}^{\theta}\right]$ are the coefficients of the thermodynamic factor matrix and $\left[\chi_{k j}^{\theta}\right]$ are the coefficients of the generalized susceptibility matrix defined as: $\left[\chi_{k j}^{\theta}\right]=\left[\Theta_{k j}^{\theta}\right]^{-1}$

\begin{tabular}{ccc}
\hline Functional & Helmholtz free energy $\mathcal{F}$ & Grand-potential $\Omega$ \\
\hline Bulk energy & $G_{m}^{\theta}\left(X_{B}^{\theta}, X_{C}^{\theta}\right)$ & $\Omega_{m}^{\theta}\left(\tilde{\mu}_{B}, \tilde{\mu}_{C}\right)$ \\
First derivatives & $\tilde{\mu}_{B}^{\theta}\left(X_{B}^{\theta}, X_{C}^{\theta}\right), \tilde{\mu}_{C}^{\theta}\left(X_{B}^{\theta}, X_{C}^{\theta}\right)$ & $X_{B}^{\theta}\left(\tilde{\mu}_{B}, \tilde{\mu}_{C}\right), X_{C}^{\theta}\left(\tilde{\mu}_{B}, \tilde{\mu}_{C}\right)$ \\
Second derivatives & $\Theta_{B B}^{\theta}, \Theta_{B C}^{\theta}, \Theta_{C C}^{\theta}$ & $\chi_{B B}^{\theta}, \chi_{B C}^{\theta}, \chi_{C C}^{\theta}$ \\
\hline
\end{tabular}




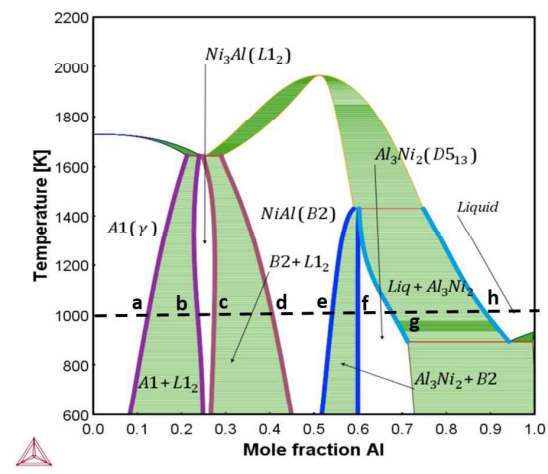

(a)

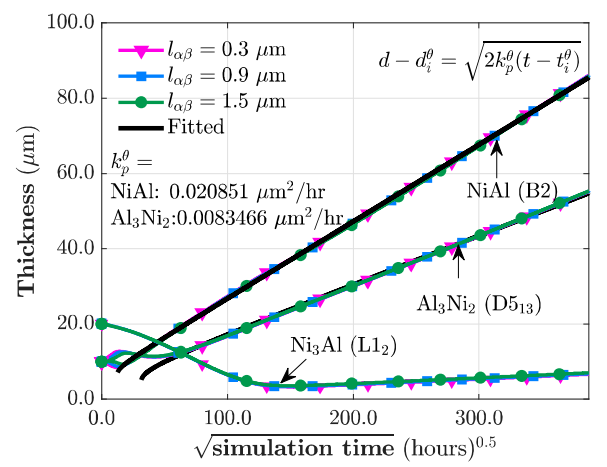

(c)

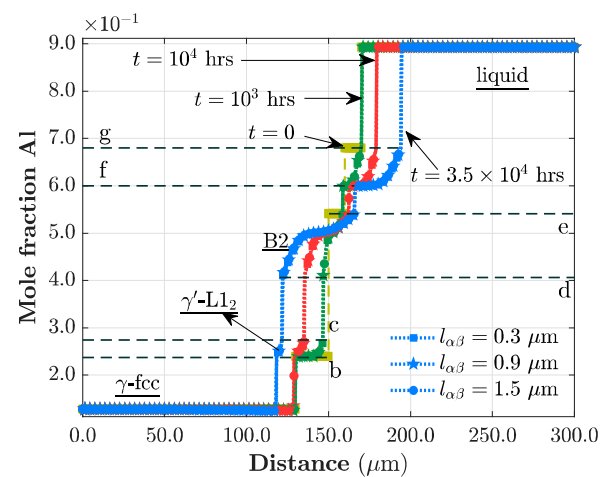

(b)

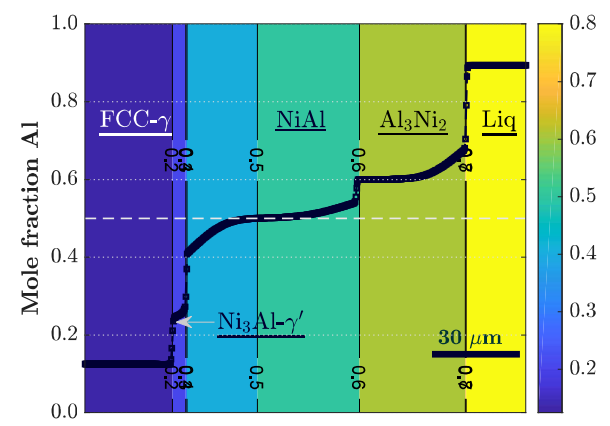

(d)

Fig. 1. a) Ni-Al phase diagram and the Al-mole fractions in the five equilibrium phases (marked a to $h$ ) at $1000 \mathrm{~K}$. For three different interface widths $l_{\alpha \beta}$ b) time evolution of simulated Al-mole fraction profiles as a function of length. In Fig.1b, the figure legend indicates this for only $t=3.5 \mathrm{e} 4 \mathrm{hrs}$, but profiles calculated at all successive time steps are superimposed. The dashed horizontal lines superimposed on Fig.1b indicate the local equilibrium Al-mole fractions (points b to g) at the two interfaces, c) the variation in the thickness of the three intermetallic phases $\mathrm{Ni}_{3} \mathrm{Al}, \mathrm{NiAl}$, and $\mathrm{Al}_{3} \mathrm{Ni}_{2}$ as a function of square root of time together with the fitted parabolic growth law, and d) two-dimensional simulation of a Ni-Al diffusion couple with a domain size of $150 \times 30 \mu \mathrm{m}^{2}$. In Fig.1d, a line profile of $\mathrm{Al}$ mole fraction calculated at $y=15 \mu \mathrm{m}$ is superimposed on the microstructure. 


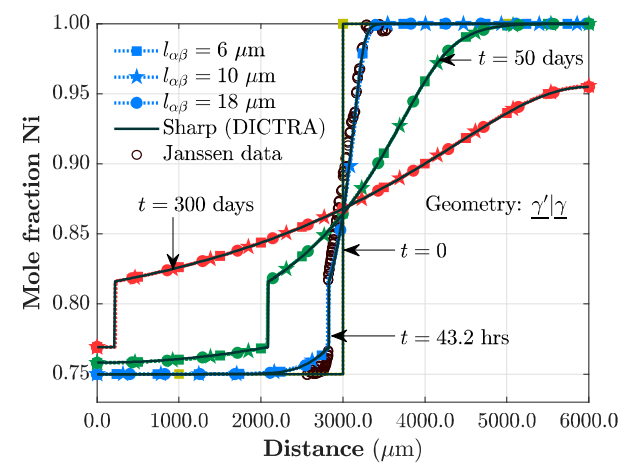

(a)

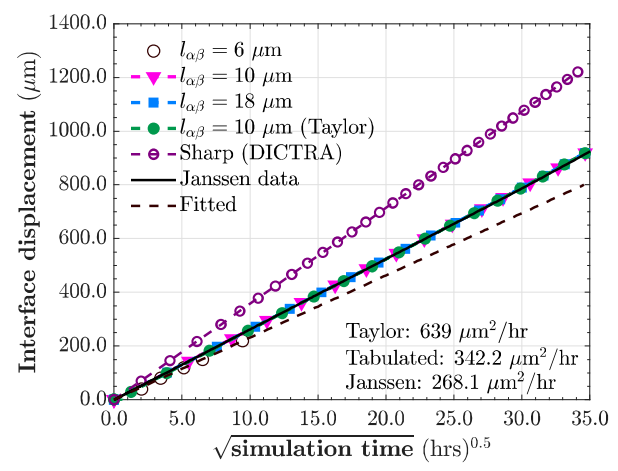

(b)

Fig. 2. For three different interface widths $l_{\alpha \beta}$ a) the evolution of the Ni-mole fraction profiles as a function of length, and b) the variation in the $\gamma-\gamma^{\prime}$ phase boundary as a function of square root of time. The experimental data from Janssen [50] and the DICTRA results are superimposed in Figs.2a and 2b. The experimentally measured Ni-mole fraction data is shown at $43.2 \mathrm{hrs}$. However, the DICTRA and phase-field simulations are calculated and compared at 50 and 300 days. 


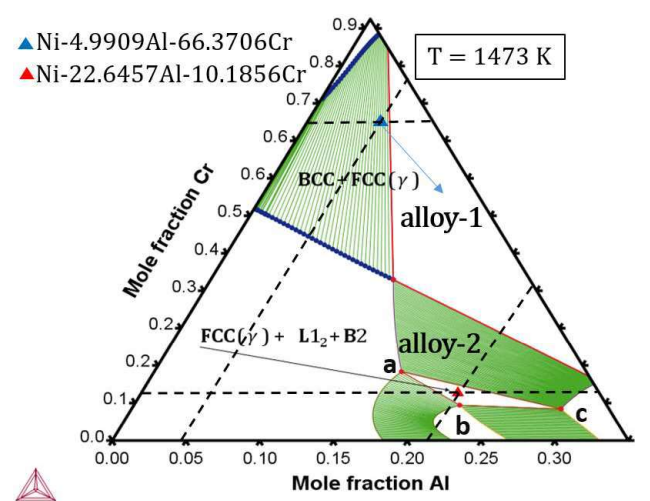

(a)

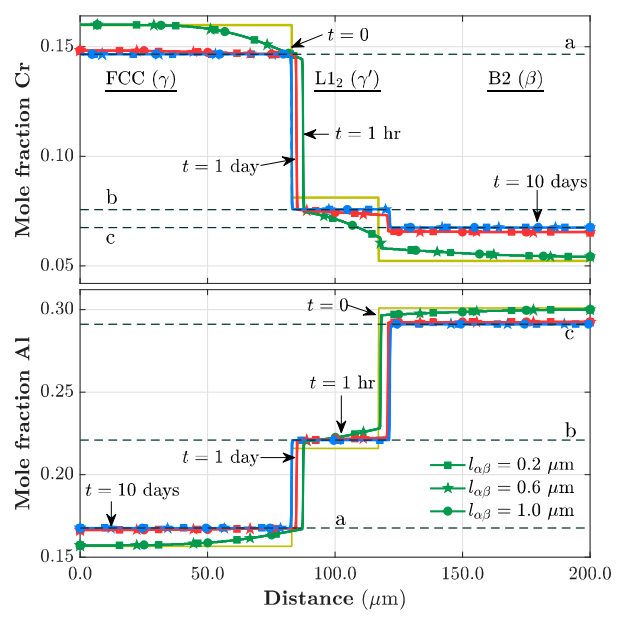

(c)

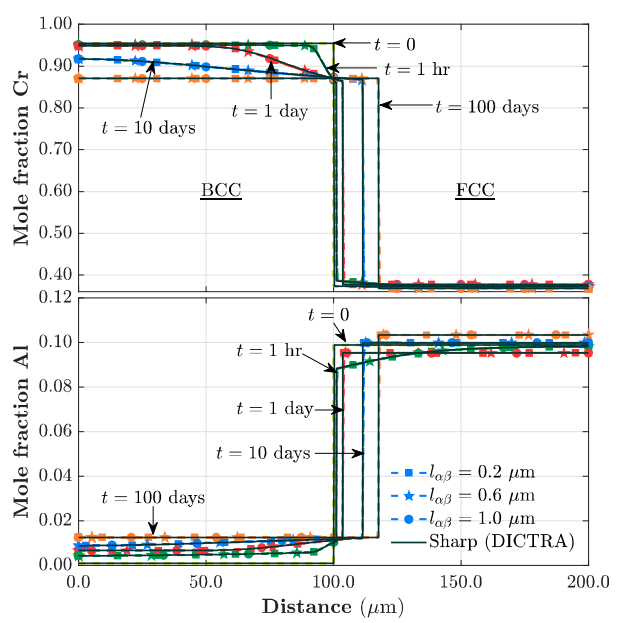

(b)
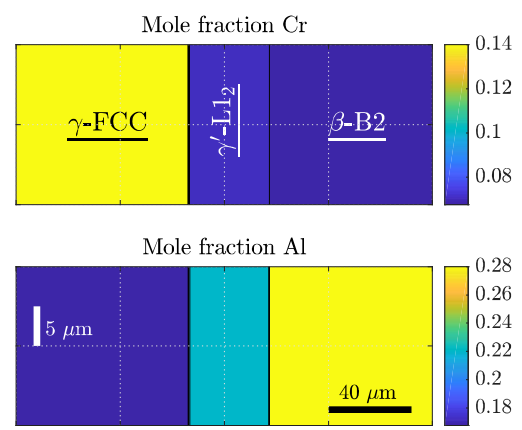

(d)

Fig. 3. a) Isothermal section and nominal compositions (in mol\%) of a twophase (T1) and a three-phase (T2) ternary Ni-Al-Cr alloys at $1473 \mathrm{~K}$. For T2, the equilibrium mole fractions in the coexisting three equilibrium phases, $\gamma$-fcc/ $/ \mathrm{L} 1_{2} / \mathrm{B} 2$, are marked by points a/b/c. Simulated time evolution of $\mathrm{Cr}$ and $\mathrm{Al}$ mole fraction profiles at $1473 \mathrm{~K}$ in a) Ni-4.9909Al-63.3706Cr (T1) alloy, and b) Ni-22.6457Al$10.1856 \mathrm{Cr}$ (T2) alloy, as a function of length for three different interface thickness $l_{\alpha \beta}$. For T1 alloy, the mole fraction profiles calculated from DICTRA are superimposed on Fig.3b. Notice that the figure legends indicates this for only one time step ( $t=10$ days in Fig.3b and $t=1 \mathrm{hr}$ in Fig.3c), but profiles calculated by varying interface thickness at each time step are superimposed. In Fig.3c, the equilibrium mole fractions of $\mathrm{Cr}$ and $\mathrm{Al}$ in $\gamma$-fcc, $\gamma^{\prime}-\mathrm{L}_{2}$ and $\mathrm{B} 2$ phases (points $\mathrm{a}, \mathrm{b}$ and $\mathrm{c}$ in Fig.3a) are marked as dashed horizontal lines. d) A two dimensional $\left(200 \times 20 \mu m^{2}\right)$ simulated micrograph of $\mathrm{Al}$ and $\mathrm{Cr}$ mole fraction fields in alloy $\mathrm{T} 2$. 


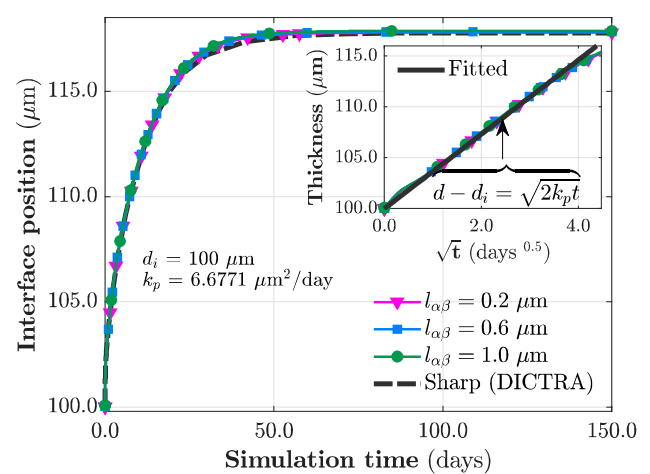

(a)

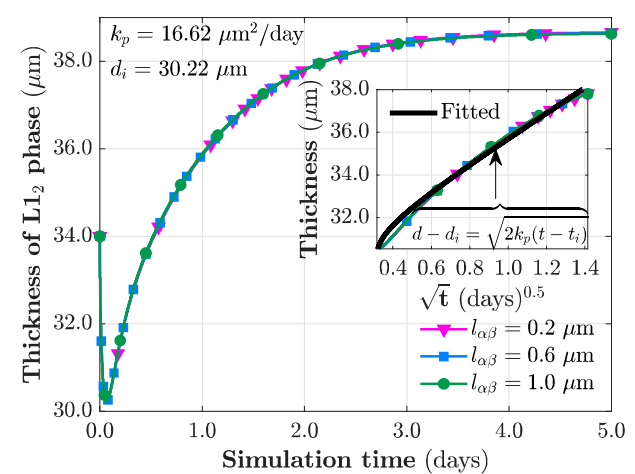

(b)

Fig. 4. For three different choice of interface widths $l_{\alpha \beta}$ a) the variation in the fcc-bcc interface position in a Ni-4.9909Al-63.3706Cr (T1) alloy, and b) the layer width of the $\mathrm{L}_{2}$ phase in a Ni-22.6457Al-10.1856Cr (T2) alloy, as functions of simulation time. The sharp interface position calculated using DICTRA for alloy $\mathrm{T} 1$ is superimposed on Fig.4a. In the insets in Figs.4a and 4b, this variation in the thickness of the bcc and $\mathrm{L}_{2}$ phases are fitted with a parabolic growth law. 


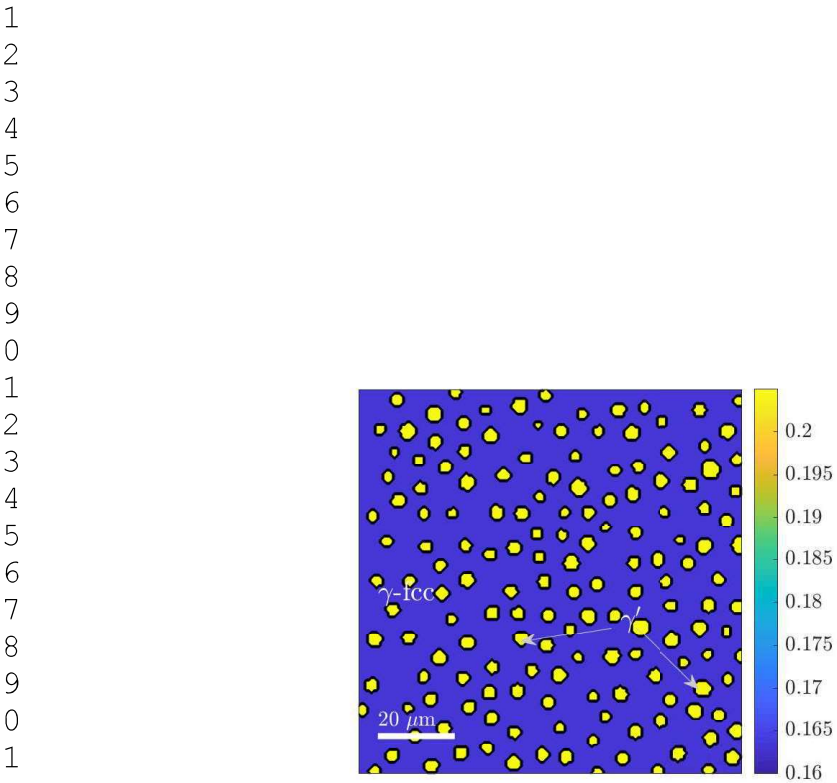

(a)

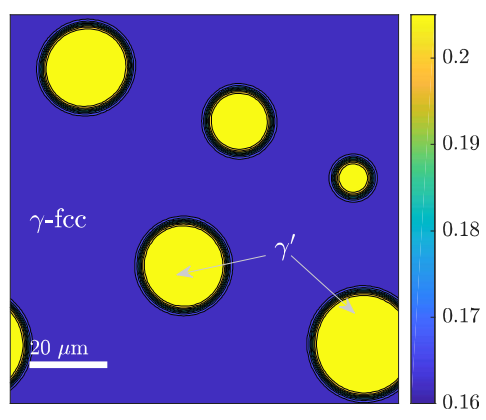

(c)

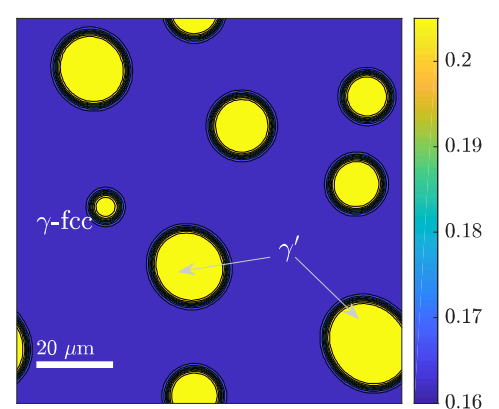

(b)

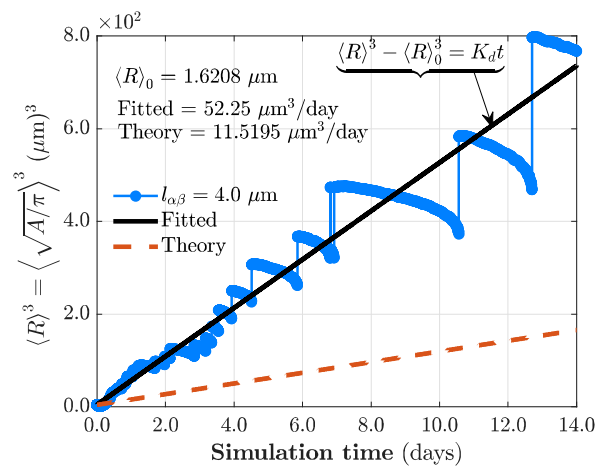

(d)

Fig. 5. Simulated Al mole fraction field of a $\gamma / \gamma^{\prime} \mathrm{Al}-\mathrm{Cr}-\mathrm{Ni}$ alloy at time a) $t=0$, b) $t=5.9916$ days, and c) $t=14$ days. d) The variation of the cube of the mean precipitate radius $\langle R(t)\rangle$ as a function of time. The domain size is $100 \times 100 \mu \mathrm{m}^{2}$, and periodic boundary conditions are employed. The theoretical and fitted growth constants are superimposed on Fig.5d. At time $t=0$, the microstructure consisted of a distribution of 160 precipitates having a mean radius of $\langle R\rangle_{0}=1.6028 \mu \mathrm{m}$. To calculate the theoretical value, the property coefficient is first determined using Eq.(25) in Ref. [52], and then multiplied by a geometric factor of (4/9). 


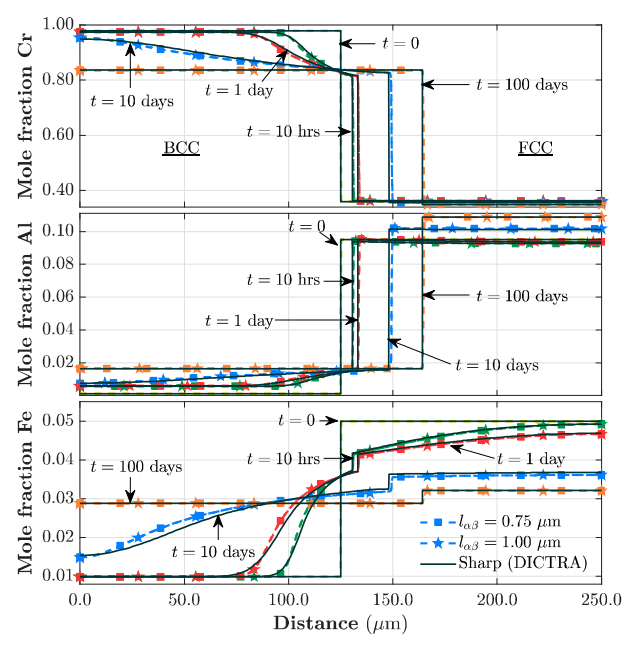

(a)

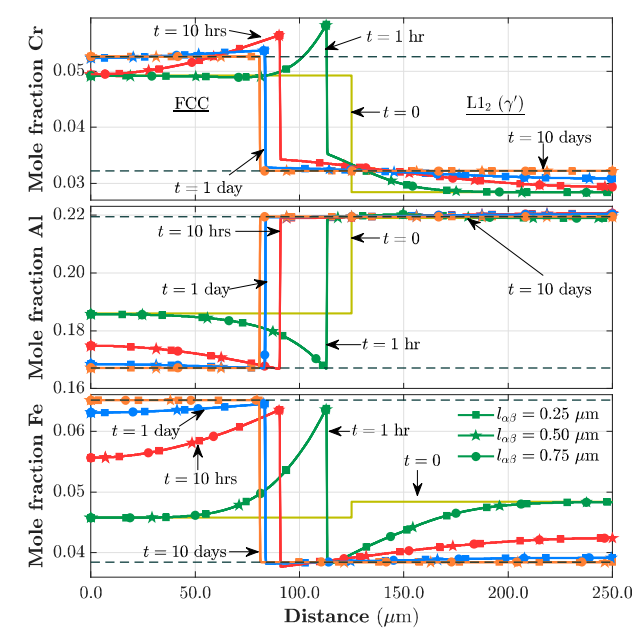

(b)

Fig. 6. Simulated time evolution of $\mathrm{Cr}, \mathrm{Al}$ and $\mathrm{Fe}$ mole fraction profiles at $1473 \mathrm{~K}$ in a) Ni-4.8095Al-66.9577Cr-2.9959Fe (Q1) alloy and b) Ni-20.25Al-3.88Cr-4.71Fe (Q2) alloy, as a function of length for three different interface thickness $l_{\alpha \beta}$. For alloy Q1, the mole fraction profiles calculated in DICTRA are superimposed on Fig.6a. Although the figure legends indicates this for only one time step $(t=10$ days in Fig.6a and $t=1 \mathrm{hr}$ in Fig.6b), but profiles calculated by varying interface thickness at each time step are superimposed. In Fig.6b, the dashed horizontal lines refer to the equilibrium solute mole fractions in alloy Q2. 


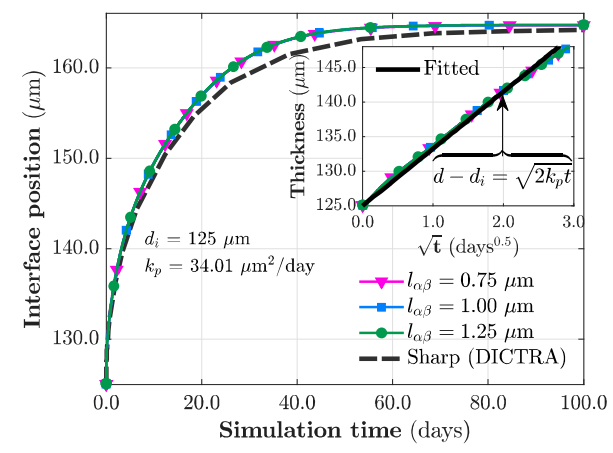

(a)

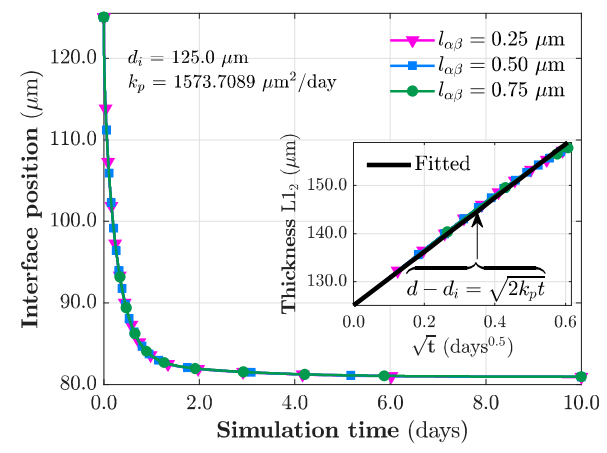

(b)

Fig. 7. For three different interface thickness $l_{\alpha \beta}$ the simulated a) fcc-bcc interface position in Q1 alloy, and b) fcc- $\mathrm{L}_{2}$ in Q2 alloy, as a function of simulation time. The position of the fcc-bcc phase boundary calculated using DICTRA for Q1 alloy is superimposed on Fig.7a. In the insets in Figs.7a and 7b, the variation in the thickness of the bcc and $\mathrm{L}_{2}$ phases are fitted to a parabolic growth law. The growth constant $k_{p}$ and the initial size $d_{i}$ for bcc and $\mathrm{L}_{2}$ calculated after fitting are superimposed on Figs.7a and 7b. 


\section{Appendix A}

\section{Derivation of diffusion equations}

To obtain the diffusion equations in terms of mole fractions, we start by differentiating the relation $\sum_{\sigma=1}^{p} h_{\sigma}(\boldsymbol{\phi})=1$

$$
\sum_{\sigma=1}^{p} \frac{\partial h_{\sigma}(\boldsymbol{\phi})}{\partial \phi_{\theta}}=0 \Longrightarrow \frac{\partial h_{\theta}(\boldsymbol{\phi})}{\partial \phi_{\theta}}=-\sum_{\substack{\sigma=1 \\ \sigma \neq \theta}}^{p-1} \frac{\partial h_{\sigma}(\boldsymbol{\phi})}{\partial \phi_{\theta}}
$$

Since the overall solute mole fraction $X_{k}(\tilde{\boldsymbol{\mu}}(\mathbf{x}, t), \boldsymbol{\phi}(\mathbf{x}, t))$ is a function of both the solute diffusion potentials and the phase-field variables, then its gradient can be expressed as

$$
\sum_{j=1}^{n-1} \frac{\partial X_{k}(\tilde{\boldsymbol{\mu}}, \boldsymbol{\phi})}{\partial \tilde{\mu}_{j}} \nabla \tilde{\mu}_{j}(\mathbf{x}, t)=\nabla X_{k}(\mathbf{x}, t)-\sum_{\theta=1}^{p} \frac{\partial X_{k}(\tilde{\boldsymbol{\mu}}, \boldsymbol{\phi})}{\partial \phi_{\theta}} \nabla \phi_{\theta}(\mathbf{x}, t)
$$

To evaluate $\partial X_{k}(\tilde{\boldsymbol{\mu}}, \boldsymbol{\phi}) / \partial \phi_{\theta}$ in Eq. (A.2), differentiate Eq. (7) with respect to the phase-field variable $\phi_{\theta}$ to obtain

$$
\frac{\partial X_{k}(\tilde{\boldsymbol{\mu}}, \boldsymbol{\phi})}{\partial \phi_{\theta}}=\sum_{\sigma=1}^{p} \frac{\partial h_{\sigma}(\boldsymbol{\phi})}{\partial \phi_{\theta}} X_{k}^{\sigma}(\tilde{\boldsymbol{\mu}})
$$

Using relation (A.1), Eq. (A.3) can be rewritten as follows:

$$
\frac{\partial X_{k}(\tilde{\boldsymbol{\mu}}, \boldsymbol{\phi})}{\partial \phi_{\theta}}=\sum_{\substack{\sigma=1 \\ \sigma \neq \theta}}^{p-1} \frac{\partial h_{\sigma}(\phi)}{\partial \phi_{\theta}}\left[X_{k}^{\sigma}(\tilde{\boldsymbol{\mu}})-X_{k}^{\theta}(\tilde{\boldsymbol{\mu}})\right]
$$


Substituting Eq. (A.4) in Eq. (A.2), and then multiplying both sides of the equation by the inverse matrix $\left[\partial X_{r} / \partial \tilde{\mu}_{k}\right]^{-1}$, we get:

$$
\nabla \tilde{\mu}_{r}(\mathbf{x}, t)=\sum_{k=1}^{n-1}\left[\frac{\partial X_{r}}{\partial \tilde{\mu}_{k}}\right]^{-1}\left(\nabla X_{k}-\sum_{\theta=1}^{p} \sum_{\substack{\sigma=1 \\ \sigma \neq \theta}}^{p-1} \frac{\partial h_{\sigma}}{\partial \phi_{\theta}}\left[X_{k}^{\sigma}-X_{k}^{\theta}\right] \nabla \phi_{\theta}\right)
$$

Finally, substituting Eq. (A.5) in Eq. (10) and using the relation between molar density and mole fraction, i.e., $c_{k}=X_{k} V_{m}$, yields Eq. (11).

Next, we derive the diffusion equations in terms of diffusion potentials, as introduced in [13], [15]. In this derivation, the difference is that instead of taking the gradient of the overall mole fraction $X_{k}(\tilde{\boldsymbol{\mu}}(\mathbf{x}, t), \boldsymbol{\phi}(\mathbf{x}, t))$, its total time derivative $\partial X_{k} / \partial t$ is evaluated, i.e.,

$$
\frac{\partial X_{k}(\mathbf{x}, t)}{\partial t}=\sum_{j=1}^{n-1} \frac{\partial X_{k}(\tilde{\boldsymbol{\mu}}, \boldsymbol{\phi})}{\partial \tilde{\mu}_{j}} \frac{\partial \tilde{\mu}_{j}}{\partial t}+\sum_{\theta=1}^{p} \frac{\partial X_{k}(\tilde{\boldsymbol{\mu}}, \boldsymbol{\phi})}{\partial \phi_{\theta}} \frac{\partial \phi_{\theta}}{\partial t}
$$

Using Eqs. (A.4), (10) \& (12), Eq. (A.6) can be expressed as:

$$
\begin{aligned}
\sum_{j=1}^{n-1}\left[\sum_{\theta=1}^{p} h_{\theta}(\phi) \chi_{k j}^{\theta}(\tilde{\boldsymbol{\mu}})\right] \frac{\partial \tilde{\mu}_{j}}{\partial t} & =\nabla\left[\sum_{j=1}^{n-1} \frac{L_{k j}^{n}(\tilde{\boldsymbol{\mu}}, \phi)}{V_{m}} \nabla \tilde{\mu}_{j}\right] \\
& -\sum_{\substack{\theta=1 \\
\theta}}^{p-1} \sum_{\substack{\sigma=1 \\
\sigma \neq \theta}}^{p} \frac{\partial h_{\sigma}}{\partial \phi_{\theta}}\left[X_{k}^{\sigma}(\tilde{\boldsymbol{\mu}})-X_{k}^{\theta}(\tilde{\boldsymbol{\mu}})\right] \frac{\partial \phi_{\theta}}{\partial t}
\end{aligned}
$$




\section{Appendix B}

\section{Equality of molar grand-potential and chemi- cal potential of dependent component}

In order to analytically verify Eqs. (1a)-(1d) in Ahmad et al. [7] for a binary alloy and Eqs. (29)-(30) in Eiken et al. [11] for a multicomponent alloy, we start by assuming constant temperature, pressure and volume. By definition, the molar Gibbs energy of a $n$-component bulk phase $\theta$ is: $G_{m}^{\theta}\left(\mathbf{X}^{\theta}\right)=\sum_{k=1}^{n} \mu_{k} X_{k}^{\theta}$, where $\mu_{k}$ is the chemical potential of an arbitrary component $k$. Using the mole fraction constraint $\sum_{k=1}^{n} X_{k}^{\theta}=1$, the definition of solute diffusion potential, $\tilde{\mu}_{k}=\left(\mu_{k}-\mu_{n}\right)$, and Eq. (6), we see that

$$
G_{m}^{\theta}(\mathbf{X}(\tilde{\boldsymbol{\mu}}))-\sum_{k=1}^{n-1} \tilde{\mu}_{k} X_{k}^{\theta}(\tilde{\boldsymbol{\mu}})=\mu_{n}^{\theta}(\tilde{\boldsymbol{\mu}})=\Omega_{m}^{\theta}(\tilde{\boldsymbol{\mu}})
$$

Figs. $8 \mathrm{a}$ and $8 \mathrm{~b}$ show the equality of molar grand-potential and chemical potential of a dependent component schematically in a molar Gibbs energy versus composition diagram in a binary A-B alloy and a ternary A-B-C alloy, respectively. In both cases, component $\mathrm{A}$ is treated as the dependent solvent component. Geometrically, the molar grand-potential, $\Omega_{m}^{\theta}(\tilde{\boldsymbol{\mu}})$, or the chemical potential of the dependent solvent component, $\mu_{A}^{\theta}(\tilde{\boldsymbol{\mu}})$, is equal to one of the intercept of the tangent line (or plane) to the molar Gibbs energy versus mole fraction(s) curve (or surface), whereas the solute diffusion potential(s) $\tilde{\boldsymbol{\mu}}$ represents the slope(s) of the tangent line (or plane in case of ternary alloy) at any given solute mole fraction(s) (Fig. B1). For the sake of clarity, we 
do not show the vertical distance below the plane of chemical potential of A (dependent component), and above the tangent plane to the Gibbs energy surface in Fig. 8b. Only a curve in the Gibbs energy surface is shown in Fig. 8b. For both substitutional and intermetallic phases, we further show this equality numerically in Fig. 8b. The phases selected to demonstrate this are: fcc \& bcc in a Cr-Ni system at $1617 \mathrm{~K}$ (Fig. 9a); $\mathrm{Ni}_{3} \mathrm{Al}\left(\mathrm{L}_{2}\right)$, fcc, $\mathrm{NiAl}$ (B2) \& liquid phases in a Ni-Al system at $1000 \mathrm{~K}$ (Fig. 9b); and fcc \& B2 in a Al-Cr-Ni system at $1473 \mathrm{~K}$ (Figs. 9c \& 9d). Fig.9 shows that the difference between the molar grand-potential and the chemical potential of the dependent component for any phase in a binary or multicomponent system is zero, and thus these two quantities are equal. 


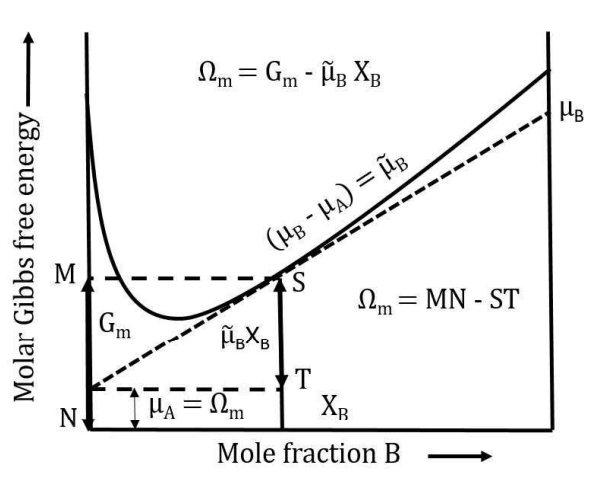

(a)

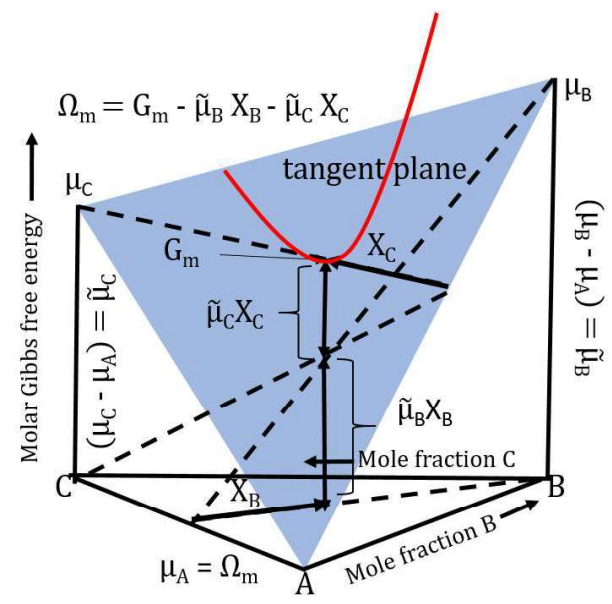

(b)

Fig. 8. The equality of molar grand-potential, $\Omega_{m}$, and the chemical potential of dependent component $\mathrm{A}, \mu_{A}$, in a molar Gibbs free energy $G_{m}$ versus mole fraction $X_{k}$ diagram is schematically shown for a) binary A-B alloy, and b) ternary A-B-C alloy. The independent diffusion potentials are here denoted by $\tilde{\mu}_{B}$ and $\tilde{\mu}_{C}$. 


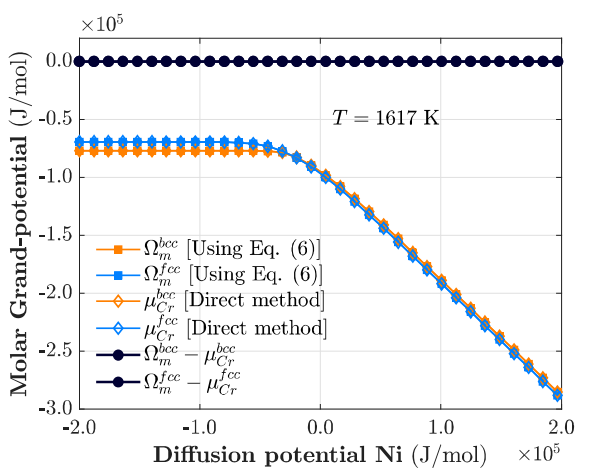

(a)

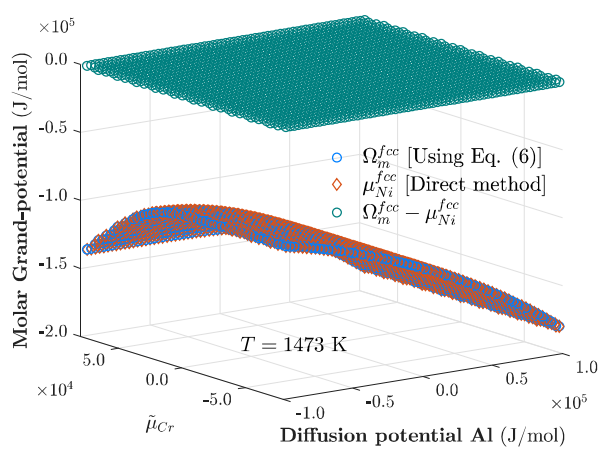

(c)

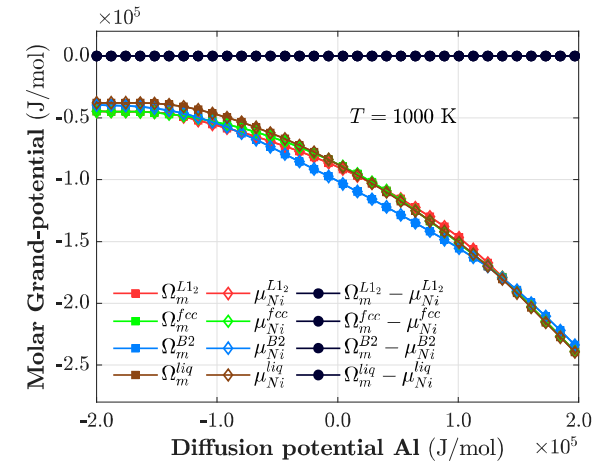

(b)

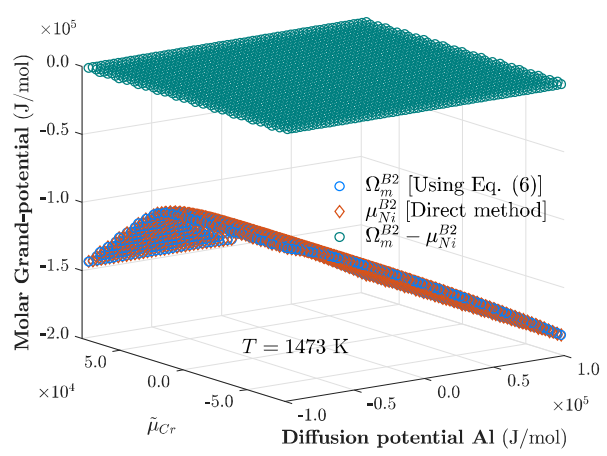

(d)

Fig. 9. Numerically calculated molar grand-potential, $\Omega_{m}$, and chemical potential of the dependent component, $\mu_{n}$, for a) two-phase binary $\mathrm{Cr}-\mathrm{Ni}$ alloy at $1617 \mathrm{~K}$, b) four-phase $\mathrm{Ni}-\mathrm{Al}$ alloy at $1000 \mathrm{~K}, \mathrm{c}$ ) ternary fcc phase in a Al-Cr-Ni alloy at $1473 \mathrm{~K}$, and d) ternary B2 phase in a Al-Cr-Ni alloy at $1473 \mathrm{~K}$. In all cases, the molar grand-potential was first calculated using Eq. (6), and then compared with the directly calculated dependent component chemical potential using an built-in command in TC-toolbox. The difference between the two quantities, i.e., $\Omega_{m}-\mu_{n}$, is superimposed in all cases. The dependent component is assumed to be $\mathrm{Cr}$ in case a), and for the remaining cases $\mathrm{Ni}$ is treated as the dependent component. 


\section{Appendix $\mathrm{C}$}

\section{Selection of diffusion potential interval and ma- terial properties}

In this section, we have detailed the diffusion potential interval(s) and the step-size taken to compute the thermodynamic or kinetic properties as discrete functions of diffusion potential(s). The step-size taken was the minimum value required to obtain quantitatively correct simulations. It is certainly possible to take a coarser step-size to compute these properties. However, in this study, we did not systematically assess the influence of the assumed step-size on the resulting phase-field simulations.

All input thermodynamic properties (i.e., the molar grand-potentials $\Omega_{m}^{\theta}$, the phase mole fractions $X_{k}^{\theta}$, and the solute susceptibility matrices $\chi_{i j}^{\theta}$ ) were precomputed using the TCNi8 database. The Onsager diffusion matrices were precomputed from the MOBNi4 and MOBFe2 databases. The latter was used to compute the Onsager matrix of the BCC phase.

\subsection{Five-phase and two-phase Ni-Al alloys}

In Fig.10a, the molar grand-potentials (or Ni-chemical potentials) and the Al-mole fractions (plotted on right y-axis) of all five equilibrium phases at $1000 \mathrm{~K}$ are plotted as a function of Al-diffusion potential. With the exception of $\mathrm{Al}_{3} \mathrm{Ni}_{2}$ phase, the $\mathrm{Al}$ phase mole fractions in all four remaining phases tend towards 1 (Al-rich) and 0 (Ni-rich) as the Al-diffusion potential varies over 
the limits $\pm 2 \mathrm{e} 5 \mathrm{~J} / \mathrm{mol}$. Thus, the three thermodynamic properties (i.e. molar grand-potential, Al-mole fraction and Al-susceptibility) of all five phases were precomputed within the interval $[-2 \mathrm{e} 5,2 \mathrm{e} 5] \mathrm{J} / \mathrm{mol}$ interval with an equal step size of $800 \mathrm{~J} / \mathrm{mol}$ (500 data points). The reason for plotting the $\mathrm{Al}_{3} \mathrm{Ni}_{2}$ phase curve over a limited interval of $[1,20] \mathrm{e} 4 \mathrm{~J} / \mathrm{mol}$ (which corresponds to a $\mathrm{Al}$ mole fraction range of $[0.6,0.94])$ is that the molar Gibbs free energy of this phase does not extend towards the Ni-rich corner of the diagram (Fig. 10b). This is due to the sublattice modelling of this phase, which is of the form $(\mathrm{Al})_{3}(\mathrm{Al}, \mathrm{Ni})_{2}(\mathrm{Va}, \mathrm{Ni})_{2}$. Since for phase-field simulations only the data within this limit was needed, this has no effect on simulated results.

For $\gamma$ and $\gamma^{\prime}$ phases, the Al-Onsager mobilities as functions of Al-diffusion potential were calculated within the above-mentioned interval, and then used in the simulations. Since the Al-mobility of $\beta$-NiAl phase as a function of Al-diffusion potential (or Al-mole fraction) could not be retrieved from the TC-Toolbox, a constant value of $1.2515 \mathrm{e}-23\left(\mathrm{~mol} \mathrm{~m} \mathrm{~m}^{2}\right) / \mathrm{J}_{\mathrm{s}}$ was used. This value was calculated from ThermoCalc's graphical user interface at a overall mole fraction of $\mathrm{Ni}-52.647 \% \mathrm{Al}(\mathrm{mol} \%)$. The Al-mobility in $\mathrm{Al}_{3} \mathrm{Ni}_{2}$ phase was set equal to $\beta-\mathrm{NiAl}$ phase, as there was no experimental data available at $1000 \mathrm{~K}$. For the liquid phase, the same constant value as for $\beta$-NiAl phase was assumed, instead of the actual values. The reason was that as the Al-mobilities in the $\beta$-NiAl and liquid phases diverged significantly $\left(L_{A l}^{N i, l i q} \approx 10^{9} L_{A l}^{N i, \beta}\right)$, we found that the simulations were not converging. The maximum divergence for which a solution was achieved was $10^{5}$, and the result is given in Supplementary material S4. Nevertheless, the proposed method can be used to simulate alloys with high contrast in Onsager mobilities. 
Two additional binary alloys with very different phase mobilities have been simulated as examples in Supplementary material S4.

Moreover, for the five-phase $\mathrm{Ni}-\mathrm{Al}$ alloy, we found that this difference in $\mathrm{Al}-$

Onsager mobilities between the $\beta$-NiAl and liquid phases was not significantly influencing the growth kinetics of the three intermediate phases: $\gamma^{\prime}, \beta$ and $\mathrm{Al}_{3} \mathrm{Ni}_{2}$. This is primarily because their growth behavior was dependent on the local equilibrium compositions and the Onsager mobilities within these phases. Since liquid is the terminal phase in this alloy, its Onsager mobility was not significantly influencing the growth rate of intermediate phases. However, the value of $\mathrm{Al}$ mobility within these three intermediate phases does influence their growth behavior.

In case of the two-phase alloy at $1473 \mathrm{~K}$, the limits of the $\mathrm{Al}$ diffusion potential interval were increased from $\pm 2 \mathrm{e} 5 \mathrm{~J} / \mathrm{mol}$ to $\pm 3 \mathrm{e} 5 \mathrm{~J} / \mathrm{mol}$ since the initial $\mathrm{Al}$ mole fraction in $\gamma$ phase is low (see subsection 3.1). Also, to ensure the same step size $(=800 \mathrm{~J} / \mathrm{mol})$ as at $1000 \mathrm{~K}$, the number of data points were increased from 500 to 750. In Fig.10a, the jump in the system Al-mole fraction (magenta) curve indicates the equilibrium compositions (see Fig.1a) of the coexisting phases, as determined by common tangent construction.

\subsection{Two-phase and three-phase Ni-Al-Cr alloys}

Based on the binary $\mathrm{Ni}-\mathrm{Al}$ case, the intervals of $\mathrm{Al}$ and $\mathrm{Cr}$ diffusion potentials were taken to vary from $[-1 \mathrm{e} 5,1 \mathrm{e} 5] \mathrm{J} / \mathrm{mol}$ with an equal step size of $400 \mathrm{~J} / \mathrm{mol}$ (2.5e5 data points). In the precomputation stage, six input thermodynamic properties (i.e., the molar grand-potential, two solute mole fractions, and 
three coefficients of the susceptibility matrix) for the ternary fcc, bcc, $\mathrm{L}_{2}$ and B2 phases were calculated as discrete functions of solute diffusion potentials. Likewise, the three independent coefficients of the Onsager mobility matrix of all but one phase, B2, were calculated in the above-mentioned intervals. Since the mobility coefficients as functions of solute diffusion potentials (or solute mole fractions) for the B2 phase could not be retrieved from the MOBNi4 database, a constant matrix $[L]^{B 2}=[0.8238,0.0552 ; 0.0552,0.2684] \mathrm{e}-17 \mathrm{~mol}$ $\mathrm{m}^{2} / \mathrm{Js}_{\mathrm{s}}$ was assumed. This components of this matrix were calculated at the equilibrium mole fraction of B2 phase using ThermoCalc's GUI mode. Fig.10c shows the precomputed molar grand-potential (or Ni-chemical potential) surfaces of fcc and bcc as functions of $\mathrm{Al}$ and $\mathrm{Cr}$ diffusion potentials.

\subsection{Two two-phase Ni-Al-Cr-Fe alloys}

Since Ni is treated as the dependent solvent component for both alloys, the molar grand-potential of quaternary fcc, bcc and $\mathrm{L}_{2}$ phases were calculated by retrieving the chemical potential of $\mathrm{Ni}$ from the TCNi8 database. In total, sixteen material properties (i.e., the molar grand-potential, three solute mole fractions, six independent coefficients of the susceptibility matrix and six independent coefficients of the Onsager matrix) of a bulk phase were first calculated and then tabulated as discrete functions of $\mathrm{Al}, \mathrm{Cr}$ and Fe diffusion potentials. For alloy Q1, the diffusion potential intervals of all solutes were taken to be $[-1 \mathrm{e} 5,1 \mathrm{e} 5] \mathrm{J} / \mathrm{mol}$ with an equal step size of $1000 \mathrm{~J} / \mathrm{mol}\left(200^{3}\right.$ data points). It is pertinent to mention here that, for alloy Q1, the precomputation time for merely collecting the thermodynamic data was nearly 7 hours, and 
the total volume of data generated was almost $8 \mathrm{~GB}$, for the mentioned resolution of the diffusion potential intervals. Therefore, to reduce the volume of the data and the precomputation time, in case of alloy Q2, the $\mathrm{Al}$ and $\mathrm{Fe}$ diffusion potential intervals were limited to a range of $[-1 \mathrm{e} 5,0] \mathrm{J} / \mathrm{mol}$ and the $\mathrm{Cr}$ diffusion potential interval was limited to $[-0.1 \mathrm{e} 5,0] \mathrm{J} / \mathrm{mol}$. Following this, the solute diffusion potential intervals were discretized into 75 equal parts $\left(75^{3}\right.$ data points). These intervals were selected after calculating the equilibrium solute diffusion potentials from ThermoCalc. The equilibrium solute diffusion potentials are $(-9.6 \mathrm{e} 4 \mathrm{Al},-0.16 \mathrm{e} 4 \mathrm{Cr},-2.1 \mathrm{e} 4 \mathrm{Fe}) \mathrm{J} / \mathrm{mol}$. This reduces the total data volume by $94.55 \%$. The disadvantage, however, is that the initial solute mole fractions cannot be taken beyond this restricted solute diffusion potential space (or the corresponding mole fraction space). 


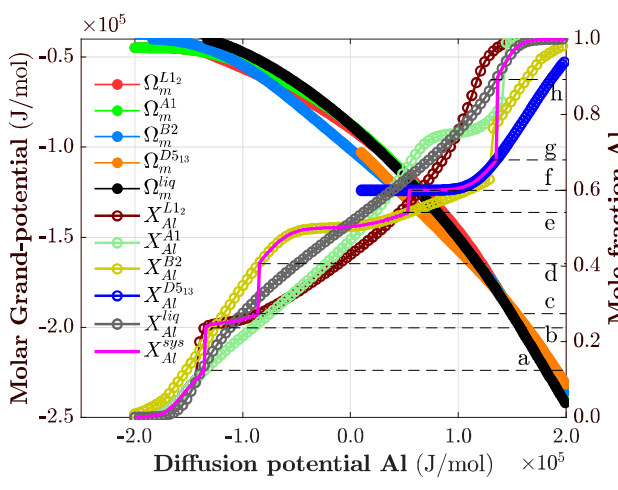

(a)

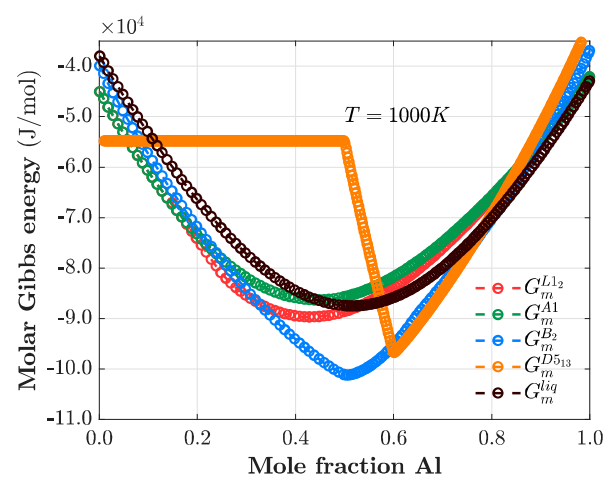

(b)

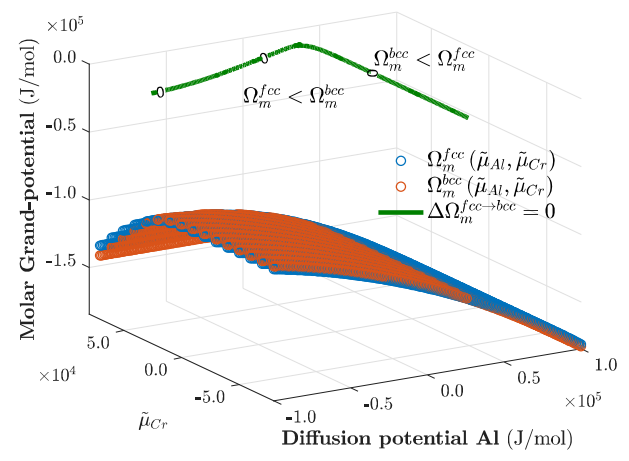

(c)

Fig. 10. For $\gamma$-fcc (A1), $\mathrm{Ni}_{3} \mathrm{Al}\left(\mathrm{L}_{2}\right), \mathrm{NiAl}(\mathrm{B} 2), \mathrm{Al}_{3} \mathrm{Ni}_{2}$ (D513) and liquid phases b) the variation in the molar grand-potentials (left $\mathrm{y}$-axis) and the $\mathrm{Al}$ phase mole fractions (right y-axis) with Al-diffusion potential at 1000K. Notice that the four jumps (or discontinuity) in the system Al-mole fraction (magenta) curve corresponds to the four two-phase regions, and the dashed horizontal lines indicate the corresponding equilibrium mole fractions, as shown in Fig.1a b) The variation in the molar Gibbs energy with $\mathrm{Al}$ mole fraction of all five equilibrium phases in $\mathrm{Ni}-\mathrm{Al}$ system at $1000 \mathrm{~K}$. c) The molar grand-potential surfaces of both fcc and bcc phases as a function of Al-diffusion potential (x-axis) and Cr-diffusion potential (y-axis) at $1473 \mathrm{~K}$. The green contour (intersection of grand-potential surfaces) indicates the ternary two-phase region in the diffusion potential space. 


\section{References}

[1] S. Gorsse, D. B. Miracle, and O. N. Senkov. "Mapping the world of complex concentrated alloys." Acta Materialia (2017); 135, pp. 177 -187 .

[2] A. Borgenstam et al. "DICTRA, a tool for simulation of diffusional transformations in alloys." Journal of Phase Equilibria (2000); 21(3), pp. 269-280.

[3] A. A. Wheeler, W. J. Boettinger, and G. B. McFadden. "Phase-field model for isothermal phase transitions in binary alloys." Phys. Rev. A (1992); 45(10), pp. 7424-7439.

[4] I. Steinbach et al. "A phase field concept for multiphase systems." Physica D: Nonlinear Phenomena (1996); 94(3), pp. 135 -147.

[5] J. Tiaden et al. "The multiphase-field model with an integrated concept for modelling solute diffusion." Physica D: Nonlinear Phenomena (1998); 115(1), pp. $73-86$.

[6] S. G. Kim, W. T. Kim, and T. Suzuki. "Interfacial compositions of solid and liquid in a phase-field model with finite interface thickness for isothermal solidification in binary alloys." Phys. Rev. E (1998); 58(3), pp. 3316-3323.

[7] N. A. Ahmad et al. "Solute trapping and solute drag in a phase-field model of rapid solidification." Phys. Rev. E (1998); 58(3), pp. 34363450. 
[8] S. G. Kim, W. T. Kim, and T. Suzuki. "Phase-field model for binary alloys." Phys. Rev. E (1999); 60(6), pp. 7186-7197.

[9] B. Echebarria et al. "Quantitative phase-field model of alloy solidification." Phys. Rev. E (2004); 70(6), p. 061604.

[10] P.-R. Cha, D.-H. Yeon, and J.-K. Yoon. "Phase-field model for multicomponent alloy solidification." Journal of Crystal Growth (2005); $274(1)$, pp. $281-293$.

[11] J. Eiken, B. Böttger, and I. Steinbach. "Multiphase-field approach for multicomponent alloys with extrapolation scheme for numerical application." Phys. Rev. E (2006); 73(6), p. 066122.

[12] S. G. Kim. "A phase-field model with antitrapping current for multicomponent alloys with arbitrary thermodynamic properties." Acta Materialia (2007); 55(13), pp. $4391-4399$.

[13] M. Plapp. "Unified derivation of phase-field models for alloy solidification from a grand-potential functional." Phys. Rev. E (2011); 84(3), pp. 031601-1-15.

[14] N. Moelans. "A quantitative and thermodynamically consistent phasefield interpolation function for multi-phase systems." Acta Materialia (2011); 59(3), pp. 1077-1086.

[15] A. Choudhury and B. Nestler. "Grand-potential formulation for multicomponent phase transformations combined with thin-interface asymptotics of the double-obstacle potential." Phys. Rev. E (2012); 85(2), pp. 021602-1-16. 
[16] M Plapp. Phase-field models. Handbook of Crystal Growth, 2nd edition, 2015, pp. 631-668.

[17] P.-R Cha, D.-H Yeon, and J.-K Yoon. "A phase field model for isothermal solidification of multicomponent alloys." Acta Materialia (2001); 49(16), pp. $3295-3307$.

[18] D.-H. Yeon, P.-R. Cha, and J.-K. Yoon. "A phase field study for ferrite-austenite transitions under para-equilibrium." Scripta Materialia (2001); 45(6), pp. $661-668$.

[19] J. Zhu et al. "Linking phase-field model to CALPHAD: application to precipitate shape evolution in Ni-base alloys." Scripta Materialia (2002); 46(5), pp. $401-406$.

[20] I. Loginova et al. "The phase-field approach and solute drag modeling of the transition to massive $\gamma \rightarrow \alpha$ transformation in binary Fe-C alloys." Acta Materialia (2003); 51(5), pp. $1327-1339$.

[21] Q. Chen et al. "Quantitative phase field modeling of diffusion-controlled precipitate growth and dissolution in Ti-Al-V." Scripta Materialia (2004); 50(4), pp. $471-476$.

[22] K Wu, Y. Chang, and Y Wang. "Simulating interdiffusion microstructures in $\mathrm{Ni}-\mathrm{Al}-\mathrm{Cr}$ diffusion couples: a phase field approach coupled with CALPHAD database." Scripta Materialia (2004); 50(8), pp. 1145 $-1150$.

[23] U. Grafe et al. "Coupling of multicomponent thermodynamic databases to a phase field model: application to solidification and solid state trans- 
formations of superalloys." Scripta Materialia (2000); 42(12), pp. 1179 $-1186$.

[24] H. Kobayashi et al. "Phase-field model for solidification of ternary alloys coupled with thermodynamic database." Scripta Materialia (2003); 48(6), pp. $689-694$.

[25] J. Zhu et al. "Quantitative interface models for simulating microstructure evolution." Acta Materialia (2004); 52(4), pp. 833 -840.

[26] R. Zhang et al. "Phase-field simulation of solidification in multicomponent alloys coupled with thermodynamic and diffusion mobility databases." Acta Materialia (2006); 54(8), pp. 2235 -2239.

[27] B. Böttger, J. Eiken, and I. Steinbach. "Phase field simulation of equiaxed solidification in technical alloys." Acta Materialia (2006); 54(10), pp. $2697-2704$.

[28] N. Moelans, B. Blanpain, and P. Wollants. "An introduction to phasefield modeling of microstructure evolution." Calphad (2008); 32(2), pp. $268-294$.

[29] N. Provatas and K. Elder. Phase-Field Methods in Materials Science and Engineering. Wiley, 2011, pp. 95-127.

[30] D. Schwen et al. "Rapid multiphase-field model development using a modular free energy based approach with automatic differentiation in MOOSE/MARMOT." Computational Materials Science (2017); 132, pp. $36-45$. 
[31] J. Heulens, B. Blanpain, and N. Moelans. "A phase field model for isothermal crystallization of oxide melts." Acta Materialia (2011); 59(5), pp. $2156-2165$.

[32] J.-O. Andersson et al. "Thermo-Calc \& DICTRA, computational tools for materials science." Calphad (2002); 26(2), pp. 273 -312.

[33] S. Schulz. "Phase-field simulations of multi-component solidification and coarsening based on thermodynamic datasets." PhD thesis. KIT, 2017, pp. 61-87.

[34] A. Choudhury et al. "Comparison of phase-field and cellular automaton models for dendritic solidification in Al-Cu alloy." Computational Materials Science (2012); 55, pp. 263 -268.

[35] D. A. Cogswell. "Quantitative phase-field modeling of dendritic electrodeposition." Phys. Rev. E (2015); 92(1), p. 011301.

[36] J. Hötzer et al. "Large scale phase-field simulations of directional ternary eutectic solidification." Acta Materialia (2015); 93, pp. 194 -204.

[37] L. Mushongera et al. "Effect of Re on directional $\gamma^{\prime}$-coarsening in commercial single crystal Ni-base superalloys: A phase field study." Acta Materialia (2015); 93, pp. $60-72$.

[38] P. Steinmetz et al. "Analytics for microstructure datasets produced by phase-field simulations." Acta Materialia (2016); 103, pp. 192 -203.

[39] M. Kellner et al. "Phase-field simulation of the microstructure evolution in the eutectic NiAl-34Cr system." Computational Materials Science (2017); 128, pp. $379-387$. 
[40] P. K. Amos, L. Mushongera, and B. Nestler. "Phase-field analysis of volume-diffusion controlled shape-instabilities in metallic systems-I: 2Dimensional plate-like structures." Computational Materials Science (2018); 144, pp. $363-373$.

[41] L. K. Aagesen et al. "Grand-potential-based phase-field model for multiple phases, grains, and chemical components." Phys. Rev. E (2018); 98(2), p. 023309.

[42] J. Hötzer et al. "Phase-field simulation of solid state sintering." Acta Materialia (2019); 164, pp. $184-195$.

[43] M. Kellner et al. "Phase-field study of eutectic colony formation in NiAl-34Cr." Acta Materialia (2020); 182, pp. 267 -277.

[44] A. Choudhury, M. Kellner, and B. Nestler. "A method for coupling the phase-field model based on a grand-potential formalism to thermodynamic databases." Current Opinion in Solid State and Materials Science (2015); 19(5), pp. $287-300$.

[45] M. Bonvalet et al. "Modeling of precipitation kinetics in multicomponent systems: Application to model superalloys." Acta Materialia (2015); 100, pp. $169-177$.

[46] Y. Guan and N. Moelans. "Influence of the solubility range of intermetallic compounds on their growth behavior in hetero-junctions." Journal of Alloys and Compounds (2015); 635, pp. $289-299$.

[47] S. M. Allen and J. W. Cahn. "A microscopic theory for antiphase boundary motion and its application to antiphase domain coarsening." Acta Metallurgica (1979); 27(6), pp. 1085 -1095. 
[48] J. Andersson and J. Ågren. "Models for numerical treatment of multicomponent diffusion in simple phases." Journal of Applied Physics (1992); 72(4), pp. 1350-1355.

[49] D. Gaston et al. "MOOSE: A parallel computational framework for coupled systems of nonlinear equations." Nuclear Engineering and Design (2009); 239(10), pp. $1768-1778$.

[50] M. M. P. Janssen. "Diffusion in the nickel-rich part of the NiAl system at $1000^{\circ} \mathrm{C}$ to $1300^{\circ} \mathrm{C} ; \mathrm{Ni}_{3} \mathrm{Al}$ layer growth, diffusion coefficients, and interface concentrations." Metallurgical Transactions (1973); 4(6), pp. 1623-1633.

[51] C. Campbell. "Assessment of the diffusion mobilites in the $\gamma^{\prime}$ and B2 phases in the Ni-Al-Cr system." Acta Materialia (2008); 56(16), pp. $4277-4290$.

[52] J. Morral and G. Purdy. "Particle coarsening in binary and multicomponent alloys." Scripta Metallurgica et Materialia (1994); 30(7), pp. 905 -908 .

[53] A. Ardell. "The effect of volume fraction on particle coarsening: theoretical considerations." Acta Metallurgica (1972); 20(1), pp. $61-71$.

[54] Y. A. Coutinho et al. "Combining thermodynamics with tensor completion techniques to enable multicomponent microstructure prediction." npj Computational Materials (Jan. 2020); 6(1), pp. 1-11. 